\title{
Revisión taxonómica de la ictiología marina de Galicia: Clase Actinopteri (Orden Acipenseriformes al Orden Scorpaeniformes)
}

\author{
Taxonomic review of Galician marine ichthyology: Class Actinopteri \\ (Order Acipenseriformes to Order Scorpaeniformes) \\ *RAFAEL BAÑón, TOÑo MAÑo \\ Grupo de Estudos do Medio Mariño (GEMM), \\ Puerto Deportivo s/n 15960 Ribeira, A Coruña, España. \\ *anoplogaster@yahoo.es; jaws.pf@gmail.com \\ (Recibido 22/10/2020; Aceptado 23/03/2021)
}

\begin{abstract}
Resumen
En este trabajo se realiza una revisión taxonómica de los peces óseos de Galicia (Clase Actinopteri) del Orden Acipenseriformes al Orden Scorpaeniformes, a través de los distintos tratados y publicaciones ictiológicas publicadas a lo largo de la historia. Se listan un total de 219 especies, de las cuales ocho se consideran dudosas, al no estar su presencia suficientemente demostrada o porque existen dudas sobre su estatus taxonómico. Una revisión de la bibliografía y nomenclatura científica nos ha permitido citar nuevas especies para Galicia y reasignar antiguas denominaciones a nuevas especies, subsanando errores de identificación de otros autores. Esta revisión permite además conocer la primera cita para cada especie en Galicia y establecer una cronología precisa del conocimiento taxonómico histórico de estos grupos de peces.
\end{abstract}

Palabras clave: Peces óseos, nomenclatura, ictiología.

\begin{abstract}
Key words: Bony fishes, nomenclature, Ichthyology.

\section{INTRODUCCIÓN}

Los peces óseos o teleósteos son el grupo más rico y diversificado de todos los vertebrados, con unas 25.000 especies en todo el mundo (alrededor del 96\% de todos los peces existentes). De manera similar, en Galicia los teleósteos comprenden alrededor del 85\% de los peces conocidos, con unas 381 especies (BAÑón \& SERRANO, 2018).
\end{abstract}

In this work, a taxonomic revision of Galician bony fishes (Class Actinopteri, from Order Acipenseriformes to Order Scorpaeniformes) is carried out through the different treaties and ichthyological publications published along history. A total of 212 species are listed, of which eight are considered doubtful, since their presence has not been sufficiently demonstrated, or because their taxonomical status is uncertain. A revision of the scientific nomenclature used so far has allowed us to reassign old names to new species, correcting identification errors by other authors. This revision also allows us to know the first citation for each species and to establish a precise chronology of the historical knowledge of the taxonomy of these groups of fishes.

Con respecto a los taxones aquí tratados, debemos destacar tres especies por la importancia económica y social que han tenido en la historia de Galicia, sobre todo en la Edad Media: la sardina Sardina pilchardus (Clupeidae), el congrio Conger conger (Congridae) y la merluza Merluccius merluccius (Merluccidae). Restos de las tres especies aparecen en los yacimientos arqueológicos de distintas épocas en 
Galicia (GonzÁLEz, 2013). Unas costumbres de Santiago de Compostela de 1133 recogidas en la Historia Compostelana muestran la existencia de un próspero mercado de pescado en el que era posible encontrar congrios, sardinas y merluzas (FERREIRA, 1998). Las tres especies figuran como parte de los pagos o diezmos que los habitantes de las poblaciones costeras hacían a los monasterios del interior (FIDALGO, 1998), y los procesos de conservación como el salado, el ahumado o el secado, permitieron su exportación a numerosas localidades por toda España (Juega Puig, 2012). Así lo atestiguan por ejemplo SARMIENTo (1762-1766) y SAÑEZ REGUART (1796), quienes mencionan en sus escritos las capturas de congrio en las costas de Galicia, así como su secado para su exportación a «Madrid, y otros parages del Reyno, de que resulta un lucro considerable»[...] «es sumamente copiosa [la pesca del congrio] en los Puertos de Cangas, Aldán, Canto de la Area, Groves [sic], etc., pero con singularidad en Puerto Marín de donde se surten los Monasterios de Religiosos de las Ordenes de San Bernardo y San Benito y otros muchos de aquel reyno» (SÁÑEz Reguart, 1796)

El principal objetivo del presente estudio es hacer una revisión taxonómica de la bibliografía y nomenclatura científica utilizada, validando la identificación de cada especie y la evolución histórica de este conocimiento.

\section{MATERIAL Y MÉTODOS}

El listado faunístico ha sido elaborado recopilando las especies listadas históricamente en tratados, publicaciones científicas y divulgativas sobre peces de Galicia. La nomenclatura científica se ha revisado según Worms (2020) y Fricke et al. (2020) salvo excepciones. La relación se presenta en orden filogenético, siguiendo el orden de clasificación propuesto por CARNEIRO et al. (2019). Para la nomenclatura de las familias se ha seguido la propuesta por VAN DER LAAN et al. (2014). En los casos en que no figura el autor del nombre científico, como sucede en López SEOANE (1866), se ha asignado el nombre válido que recoge la literatura científica. Las variaciones no significativas en la grafía de los nombres científicos se recogen entre corchetes a continuación del nombre del autor. Se consideran "errores de identificación" aquellas especies que han sido nombradas originalmente con una nomenclatura que no corresponde ni a su nombre válido ni a ninguno de sus sinónimos conocidos. Las referencias bibliográficas se corresponden con todas las presentes en los siglos XVIII y XIX y una selección de las posteriores, sobre todo las que se refieren a revisiones y actualizaciones faunísticas.

\section{RESULTADOS}

\section{Superclass OSTEICHTHYES Clase ACTINOPTERI}

Orden ACIPENSERIFORMES Berg, 1940 Familia ACIPENSERIDAE Bonaparte, 1831

Acipenser sturio Linnaeus, 1758

Acipenser sturio Linnaeus, 1758: PAZ Graells (1870)

Comentarios: Especie extinguida de nuestras costas, si bien antes solían cogerse en la embocadura de las rías (PAZ Graells, 1870).

Orden NOTACANTHIFORMES Berg, 1947 Familia HALOSAURIDAE Günther, 1868

Halosaurus ovenii Johnson, 1864

Halosaurus ovenii Johnson, 1864: PIÑEIRO et al. (1996); BAÑóN et al. (1997); BAÑóN et al. (2010), BAÑón et al. (2016a,b), BAÑóN \& Serrano (2018)

Halosauropsis macrochir (Günther, 1878)

Aldrovandia macrochir (Günther, 1878): DE BuEN (1935a)

Halosauropsis macrochir (Günther, 1878): Solórzano et al. (1988); Rodríguez VillanUEVA \& VÁzQuez (1995); BAÑón et al. (2010), BAÑóN et al. (2016a,b); BARros GArcía et al. (2016a); Bañón \& SERrano (2018)

Aldrovandia phalacra (Vaillant, 1888)

Halosaurus phalacrus Vaillant, 1888: DE BuEN (1935a)

Aldrovandia phalacra (Vaillant, 1888): SoLÓRZANo et al. (1988); RodríGUEZ VILlANUEVA \& VÁzQUEZ (1995); BAÑón et al. (2010), BAÑóN et al. (2016a,b); Barros GARcía et al. (2016); Bañón \& SERrano (2018) 
Aldrovandia affinis (Günther, 1877)

Aldrovandia affinis (Günther, 1877): BAÑóN et al. (2016a,b); BArros GArcía et al. (2016a); BAÑón \& SERRANo (2018)

Aldrovandia oleosa Sulak, 1977

Aldrovandia oleosa Sulak, 1977: BAÑón et al. (2016a,b); BARros GARCíA et al. (2016a); BAÑóN \& Serrano (2018)

Familia NOTACANTHIDAE Rafinesque, 1810

Notacanthus bonaparte Risso, 1840

Notacanthus bonapartei Risso, 1840: SolóRZANO et al. (1983, 1988); PIÑEIRO et al. (1996); Rodríguez Villanueva \& VÁzQuez (1995)

Notacanthus bonaparte Risso, 1840: BAÑóN et al. (2016a,b); BARros GARCÍA et al. (2016a); Bañón \& Serrano (2018); Barros García et al. (2020)

A

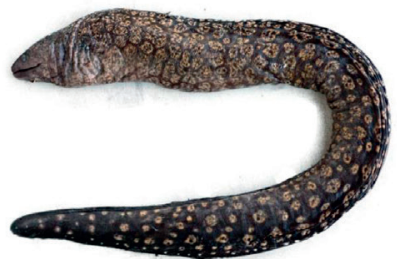

C

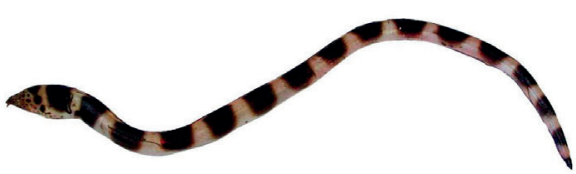

$\mathbf{E}$

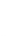

Figura 1. Peces Anguiliformes presentes en Galicia. (A) Muraena helena Linnaeus, 1758 (Muraenidae). (B) Ophisurus serpens (Linnaeus, 1758) (Ophichthidae). (C) Pisodonophis semicinctus (Richardson, 1848) (Ophichthidae). (D) Pseudophichthys splendens (Congridae). (E) Nessorhamphus ingolfianus (Schmidt, 1912) (Derichthyidae). (F) Nettastoma melanura Rafinesque, 1810 (Nettastomatidae).

Figure 1. Anguilliform fishes in Galicia. (A) Muraena helena Linnaeus, 1758 (Muraenidae). (B) Ophisurus serpens (Linnaeus, 1758) (Ophichthidae). (C) Pisodonophis semicinctus (Richardson, 1848) (Ophichthidae). (D) Pseudophichthys splendens (Congridae). (E) Nessorhamphus ingolfianus (Schmidt, 1912) (Derichthyidae). (F) Nettastoma melanura Rafinesque, 1810 (Nettastomatidae).
Notacanthus chemnitzii Bloch, 1788

Notacanthus chemnitzii Bloch, 1788: SolóRZANO et al. (1988); RodRíguez VILlanUEVA \& VÁzQUEZ (1995); BAÑóN et al. (2010); BAÑóN et al. (2016a,b); BARros GARCíA et al. (2016a); Bañón \& SERRANo (2018)

Polyacantonotus rissoanus (De Filippi \& Vérany, 1857)

Polyacantonotus rissoanus (De Filippi \& Vérany, 1857): BAÑón et al. (2016a,b); BARRos García et al. (2016a); Bañón \& SerRano(2018)

Orden ANGUILLIFORMES Berg, 1940

Familia SYNAPHOBRANCHIDAE Johnson, 1862

Synaphobranchus kaupii Johnson, 1862

Synaphobranchus pinnatus Gronow (1854):

De Buen (1935a)

B

D

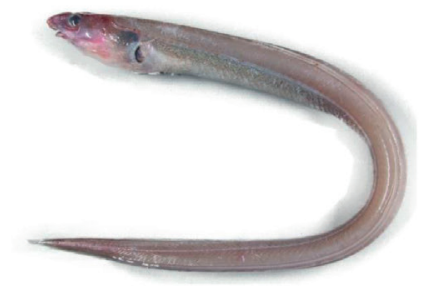

F
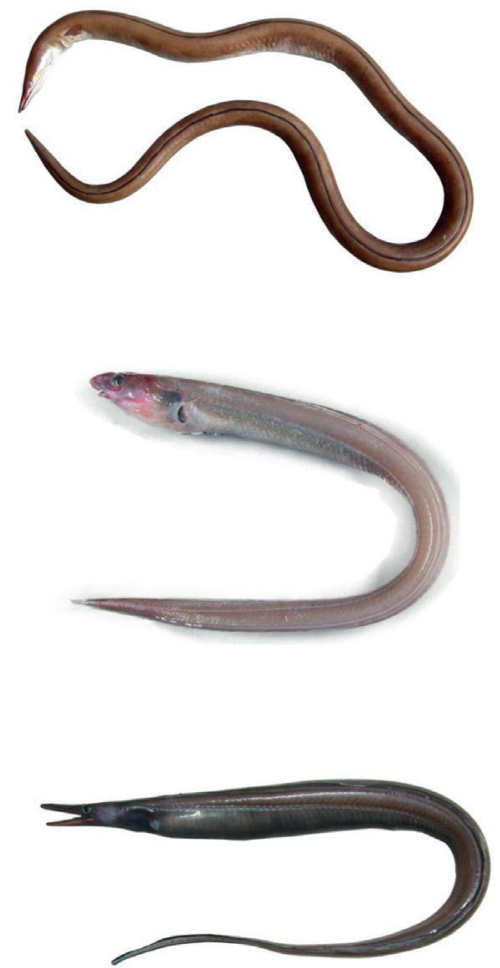
Synaphobranchus kaupii Johnson, 1862: Solórzano et al. (1988); Rodríguez VillaNUEVA \& VÁzQuez (1995); PiÑEIRo et al. (1996); BAÑóN et al. (2010); BAÑón \& SERRANO (2018)

Familia ANGUILLIDAE Rafinesque, 1810

Anguilla anguilla (Linnaeus, 1758)

Muraena anguilla Linnaeus, 1758: CORNIDE (1788); LozAno Rey (1947)

Anguilla vulgaris Rafinesque, 1810: Anguilla latirostris Risso, 1827: López SEOANe (1866)

Anguilla mediorostris Risso, 1827: PAZ Graells (1870) [Anguilla medirostris]

Anguilla anguilla (Linnaeus, 1758): DE Buen (1935a); Solórzano et al. (1983, 1988); Rodríguez Villanueva \& VÁzquez (1995); BAÑón et al. (2010); BAÑón \& SERRANo (2018)

Familia MURAENIDAE Rafinesque, 1815

Muraena helena Linnaeus, 1758 (Fig. 1A)

Muraena ophis Linnaeus, 1758: CORNIDE (1788)- error de identificación

Muraenophis helena (Linnaeus, 1758): LoZANO REY (1947) [Murenophis helena]

Muraena helena Linnaeus, 1758: LóPEz SeOANe (1866) [Muroena helena]; PAz Graells (1870) [Murena helena]; De BuEN (1919) [Murena helena]; De Buen (1935a); Solórzano et al. (1983, 1988); Rodríguez Villanueva \& VÁzquez (1995); BAÑón et al. (2010); BAÑóN \& Serrano (2018)

Comentarios: Si bien la descripción dada por CORNIDE (1788) se corresponde con la morena $M$. helena L., la denominación $M$. ophis L. es un sinónimo de Ophichthus ophis (Linnaeus, 1758) (Ophichthidae) y se corresponde por tanto con otra especie.

\section{Familia OPHICHTHIDAE Günther, 1870} 1B)

Ophisurus serpens (Linnaeus, 1758) (Fig.

Ophisurus serpens (Linnaeus, 1758): LóPEz SeOANe (1866); BAÑón et al. (2011a) BAÑón \& Serrano (2018)

Pisodonophis semicinctus (Richardson, 1848) (Fig. 1C)
Pisodonophis semicinctus (Richardson, 1848): BAÑón et al. (2002); BAÑón et al. (2010); Bañón \& Serrano (2018)

Comentarios: Especie conocida en Galicia por un solo ejemplar de $71 \mathrm{~cm}$ capturado el 20 de Julio de 2001 en A Guarda (BAÑón et al., 2002)

Familia CONGRIDAE Kaup, 1856

Conger conger (Linnaeus, 1758)

Muraena conger Linnaeus, 1758: CORNIDE (1788)

Conger vulgaris Yarrell, 1832: LóPez SEOANE (1866); Paz Graells (1870)

Conger niger (Risso, 1810): PAz Graells (1870)

Leptocephalus conger (Linnaeus, 1758): DE Buen (1935a)

Conger conger (Linnaeus, 1758): FERNÁNDEZ et al. (1978); SolóRZANo et al. (1983, 1988); FARIÑA et al. 1985; Rodríguez VillanUEVA \& VÁzQuez (1995); PiÑEIRo et al. (1996); BAÑóN et al. (2010); BAÑón et al. (2016a); BAÑÓN \& SERrano (2018)

Pseudophichthys splendens (Lea, 1913) (Fig. 1D)

Pseudophichthys splenden (Lea, 1913): BAÑón et al. (2011b); BAÑón et al. (2016a); Bañón \& Serrano (2018)

Comentarios: Especie conocida por dos ejemplares, un adulto de $31 \mathrm{~cm}$ y un juvenil de $12 \mathrm{~cm}$, ambos capturados en el banco de Galicia (BAÑóN et al. 2011b)

Familia DERICHTHYIDAE Gill, 1884

Nessorhamphus ingolfianus (Schmidt, 1912) (Fig. 1E)

Nessorhamphus ingolfianus (Schmidt, 1912): BAÑón et al. (2016a); BAÑón \& SERRANO (2018)

Familia NEMICHTHYIDAE Kaup, 1859

Nemichthys scolopaceus Richardson, 1848

Nemichthys scolopaceus Richardson, 1848: LozAno Rey (1947); Solórzano et al. (1988); Rodríguez Villanueva \& VÁzQuez (1995); PiÑeIro et al. (1996); BAÑón et al. (2010); BAÑón et al. (2016a); BAÑón \& SERRANo (2018) 
Familia SERRIVOMERIDAE Trewavas, 1932

Serrivomer beanii Gill \& Ryder, 1883

Serrivomer beanii Gill \& Ryder, 1883: PIÑEIRo et al. (1996); BAÑóN et al. (2010); BAÑóN et al. (2016a); BAÑón \& SERRANo (2018)

Familia NETTASTOMATIDAE Kaup, 1859

Nettastoma melanura Rafinesque, 1810 (Fig. 1F)

Nettastoma melanurum Rafinesque, 1810: PiñeIro et al. (1996); BAÑón et al. (2010); Bañón \& Serrano (2018)

Nettastoma melanura Rafinesque, 1810: BAÑón et al. (1997)

Facciolella oxyrhyncha (Bellotti, 1883)

Facciolella oxyrhyncha (Bellotti, 1883): BAÑón et al. (2010); BAÑón \& SERRANo (2018)

\section{Orden SACCOPHARYNGIFORMES}

Familia EURYPHARYNGIDAE Gill, 1883

Eurypharynx pelecanoides Vaillant, 1882

Eurypharynx pelecanoides Vaillant, 1882: De Buen (1935a); Lozano Rey (1947); SolóRZANo et al. (1988); RODRÍGUEZ VILLANUEVA \& VÁzQuez (1995); PiñeIro et al. (1996); BAÑóN et al. (2010); BAÑón et al. (2016a); BAÑóN \& SERrano (2018)

Saccopharynx ampullaceus (Harwood, 1827)

Saccopharynx johnsoni Bertin, 1934: DE BUEN (1936)

Orden CLUPEIFORMES Goodrich, 1909

Familia CLUPEIDAE Cuvier, 1816

Sardina pilchardus (Walbaum, 1792)

Arengus minor Cornide, 1788: CoRNIDE (1788)

Clupea harengus pilchardus Walbaum, 1792:

De Buen (1935a) [Arengus pilchardus]

Clupea sardina Cuvier, 1829: López SEOANE (1866)

Clupea harengus pilchardus (Walbaum, 1792): PAZ Graells (1870) [Clupea pilchardus], De Buen (1919) [Clupea pilchardus]

Alausa pilchardus Valenciennes, 1847: PAZ Graells (1870)
Clupanodon pilchardus Risso, 1826: PAZ Graells (1870)

Sardina pilchardus (Walbaum, 1792): LoPEZ VeIga (1978); Solórzano et al. (1983, 1988); FARiÑa et al. (1985); Rodríguez Villanueva \& VÁzQUEz (1995); BAÑóN et al. (2010); BAÑóN \& Serrano (2018)

Comentarios: Es con toda seguridad el pez más conocido y estudiado de nuestras costas, debido a la importancia comercial que siempre ha tenido en la economía gallega. RodRíGUEZ Villanueva \& VÁzquez (1995) registran 63 referencias bibliográficas hasta 1995, con diferencia la especie que más referencias aporta en su obra.

Alosa fallax (Lacepède, 1803)

Clupea finta Cuvier, 1829: López SeoAne (1866)

Alosa fallax (Lacepède, 1803): De Buen (1935a); Solórzano et al. (1988); FARIÑa et al. (1985); Rodríguez Villanueva \& VÁzQUEZ (1995); BAÑóN et al. (2010); BAÑóN \& SERrano (2018)

Alosa alosa (Linnaeus, 1758)

Clupea alosa Linnaeus, 1758: CorNIDE (1788); De Buen (1919)

Alosa communis Yarrell, 1836: LóPEz SEOANE (1866)

Alosa alosa (Linnaeus, 1758): De Buen (1935a); Lozano Rey (1947); Fernández et al. (1981) Solórzano et al. (1983, 1988); FARiÑa et al. (1985); RodrígueZ VILlanUeVA \& VÁzQuez (1995); BAÑón et al. (2010); BAÑóN \& Serrano (2018)

Clupea harengus Linnaeus, 1758

Clupea harengus Linnaeus, 1758: BAÑóN et al. (2019)

Sprattus sprattus (Linnaeus, 1758)

Clupea alosa parva Linnaeus, 1758: CORNIDE (1788) - error de identificación

Sardinella aurita Cuvier and Valenciennes, 1847: PAZ Graells (1870)- error de identificación

Clupea sprattus Linnaeus, 1758: LozANO Rey (1919, 1947); Oliver (1950) 
Spratella sprattus (Linnaeus, 1758): DE BUEN (1935a)

Sprattus sprattus (Linnaeus, 1758): LóPEz Veiga, E.C. (1976); Solórzano et al. (1983, 1988); FARIÑA et al. (1985); RodRÍGUEZ VILLANUEVA \& VÁzQUEz (1995); BAÑón et al. (2010); Bañón \& Serrano (2018)

Comentarios: CoRnIDE (1788) creía erróneamente que el espadín $S$. sprattus era la cría del sábalo $A$. alosa, por eso añade parva (pequeño) al nombre científico $C$. alosa. PAZ GrAELls (1870) asigna el nombre de la alacha Sardinella aurita Valenciennes, 1847 al espadín, creyendo que la especie del Mediterráneo y la del Atlántico eran la misma. De hecho mezcla los nombres comunes de ambas, pero $S$. aurita no está citada en Galicia.

Engraulis encrasicolus (Linnaeus, 1758)

Clupea encrasicolus Linnaeus, 1758: CoRNIDE (1788)

Engraulis encrasicholus Cuvier, 1817: LóPEZ Seoane (1866) [Engraulis encrosicholus]; PAZ Graells (1870)

Engraulis encrasicolus (Linnaeus, 1758): LoZANo Rey (1919); De Buen (1935a) [Engraulis encrassicholus]; SOLÓRZANO et al. (1983, 1988); FARIÑA et al. (1985); RodríGUEZ VILlanUEVA \& VÁzquez (1995); BAÑón et al. (2010); BAÑóN \& SerRano (2018)

\section{Orden OSMERIFORMES}

Familia ARGENTINIDAE Bonaparte, 1846

Argentina sphyraena Linnaeus, 1758

Argentina sphyraena Linnaeus, 1758: FerNÁNDEZ et al. (1976) [Argentina sphiraena]; FARIÑa et al. (1985); SOlÓRZANo et al. (1983, 1988); FARIÑA et al. (1985); RodRÍGUEZ VILLANUEVA \& VÁzQUEZ (1995); BAÑón et al. (2010); BAÑón \& SERrano (2018)

Argentina silus (Ascanius, 1775)

Argentina silus (Ascanius, 1775): BAÑón et al. (2010); BAÑón \& Serrano (2018)

Familia OPISTHOPROCTIDAE Schmidt, 1918

Opisthoproctus soleatus Vaillant, 1888

Opisthoproctus soleatus Vaillant, 1888: BAÑón \& SERrano (2018)
Familia BATHYLAGIDAE Gill, 1884

Bathylagus euryops Goode \& Bean, 1896

Bathylagus elongatus Roule, 1916: De BuEN (1935a); LozAno Rey (1947)

Bathylagus euryops Goode \& Bean, 1896: Solórzano et al. (1988); Rodríguez VillaNUEVA \& VÁzQUEZ (1995); BAÑón et al. (2010); BAÑón et al. (2016a); BAÑón \& SERrano (2018)

Familia ALEPOCEPHALIDAE Bonaparte, 1846

Alepocephalus rostratus $\mathrm{R}$ isso, 1820

Alepocephalus rostratus Risso, 1820: PIÑEIRO et al. (1996); BAÑóN (2002); BAÑón et al. (2010, 2016a); BAÑón \& Serrano (2018)

Alepocephalus bairdii Goode \& Bean, 1879

Alepocephalus bairdii Goode \& Bean, 1879: PIÑEIRO et al. (1996); BAÑóN (2002); BAÑóN et al. (2010; 2016a); BAÑón \& Serrano (2018)

Alepocephalus agassizi Goode \& Bean, 1883

Alepocephalus agassizi Goode \& Bean, 1883: BAÑón \& SERRANo (2018)

Conocara macropterum (Vaillant, 1888)

Conocara macropterum (Vaillant, 1888): BAÑón et al. (2016a); BAÑón \& SERRANO (2018)

Rouleina attrita (Vaillant, 1888)

Rouleina attrita (Vaillant, 1888): BAÑóN et al. (2016a); BAÑón \& SERRANo (2018)

Xenodermichthys copei (Gill, 1884)

Xenodermichthys copei (Gill, 1884): RodRíGuez Villanueva \& Vázquez (1995); Piñeiro et al. (1996); BAÑóN (2002); BAÑón et al. (2010, 2016a); BAÑón \& SERrano (2018)

Xenodermichthys socialis (Vaillant, 1888): De Buen (1935a); LozAno Rey (1947); FARIÑA et al. (1985); SolóRzANo et al. (1988)

Familia PLATYTROCTIDAE Koefoed, 1927

Holtbyrnia macrops Maul, 1957

Holtbyrnia problematica (Parr, 1960): FARIÑA et al. (1985); SolóRZANo et al. (1988)

Holtbyrniamacrops Maul, 1957: RodRÍGUEZ VillanueVa \& VÁzQuez (1995); BAÑón et al. (2010); Bañón \& Serrano (2018) 
Comentarios: Rodríguez Villanueva \& VÁzQuez (1995) citan además Holtbyrnia anomala (Kreft, 1980) citada por ZUGMAYER en Lozano Rey (1947). Sin embargo, revisado LoZANO REY (1947), no encontramos mencionada esta especie, por lo que no se ha tenido en cuenta para el listado.

Mentodus rostratus (Günther, 1878)

Bathytroctes rostratus Günther, 1878: Zugmayer en Lozano Rey (1947)

Mentodus rostratus (Günther, 1878): SoLÓRZANO et al. (1988); BAÑón et al. (2010); BAÑón \& SERRANo (2018)

Comentarios: La cita original proviene de ZugMAYER, quien la cita frente a Coruña (LozAno Rey, 1947)

Orden SALMONIFORMES Bleeker, 1859 Familia SALMONIDAE Jarocki or Schinz, 1822

A

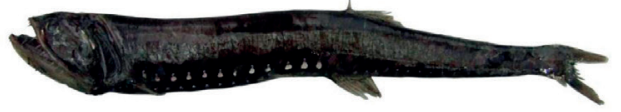

C
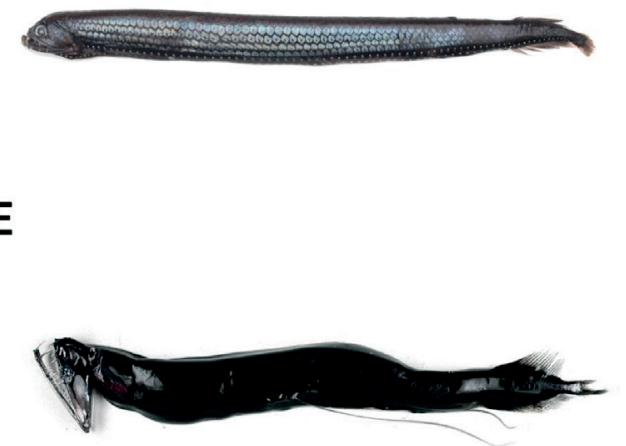

Salmo trutta Linnaeus, 1758

Salmo trutta Linnaeus, 1758: Salmo trutta (Cornide, 1788); López Seoane (1866); De Buen (1919); Solórzano et al. (1983); BAÑóN et al. (2010); BAÑón \& SERrAno (2018)

Salmo salar trutta Smith, 1895: LozANo REY (1919)

Salmo salar Linnaeus, 1758

Salmo salar Linnaeus, 1758: CoRNIDE (1788); López SeoAne (1866); De Buen (1919); LozAno Rey (1919); Solórzano et al. (1983); BAÑón et al. (2010); BAÑón \& SERRANO (2018)

Orden STOMIIFORMES Regan, 1909 Familia GONOSTOMATIDAE Cocco, 1838

Gonostoma elongatum Günther, 1878(Fig. 2A)

Gonostoma elongatum Günther, 1878: PIÑEIRO et al. (1996); BAÑón et al. (2010, 2016a); Bañón \& SERrano (2018)

B

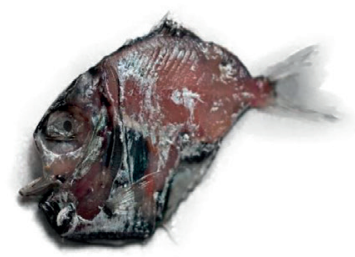

D

$\mathbf{F}$

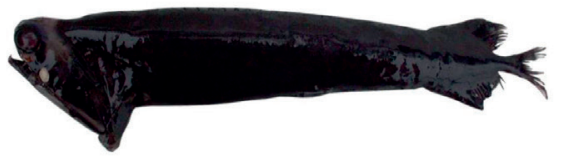

Figura 2. Peces Estomiformes presentes en Galicia. (A) Gonostoma elongatum Günther, 1878 (Gonostomatidae). (B) Sternoptyx diaphana Hermann, 1781 (Sternoptychidae). (C) Stomias boa (Risso, 1810) (Stomiidae). (D) Chauliodus sloani Bloch \& Schneider, 1801 (Stomiidae). (E) Photostomias guernei Collet, 1889 (Stomiidae). (F) Malacosteus niger Ayres, 1848 (Stomiidae).

Figure 2. Stomiform fishes in Galicia. (A) Gonostoma elongatum Günther, 1878 (Gonostomatidae). (B) Sternoptyx diaphana Hermann, 1781 (Sternoptychidae). (C) Stomias boa (Risso, 1810) (Stomiidae). (D) Chauliodus sloani Bloch \& Schneider, 1801 (Stomiidae). (E) Photostomias guernei Collet, 1889 (Stomiidae). (F) Malacosteus niger Ayres, 1848 (Stomiidae). 
Sigmops bathyphilus (Vaillant, 1884)

Gonostoma bathyphilum (Vaillant, 1884): De Buen (1935a); Zugmayer en Lozano Rey (1947); Rodríguez Villanueva \& Vázquez (1995)

Sigmops bathyphilus (Vaillant, 1884): PIÑEIRO et al. (1996); BAÑón et al. (2010, 2016a); BAÑón \& SERRANo (2018)

Cyclothone braueri Jespersen \& Tåning, 1926

Cyclothone braueri Jespersen \& Tåning, 1926: De Buen (1935a); SOlórzano et al. (1988); Rodríguez Villanueva \& VÁzQuez (1995); BAÑón et al. (2010); BAÑón \& SERRANo (2018)

Cyclothone microdon (Günther, 1878)

Cyclothone microdon (Günther, 1878): De Buen (1935a); Solórzano et al. (1988); Rodríguez Villanueva \& VÁzQuez (1995); BAÑón et al. (2010); BAÑón \& SERRANO (2018)

Cyclothone pallida Brauer, 1902

Cyclothone pallida Brauer, 1902: BAÑón et al. (2016a)

Familia STERNOPTYCHIDAE Duméril, 1805

Sternoptyx diaphana Hermann, 1781 (Fig. 2B)

Sternoptyx diaphana Hermann, 1781: DE Buen (1935a); Lozano Rey (1947); SolórzaNo et al. (1988); Rodríguez Villanueva \& VÁzQuez (1995); BAÑón et al. (2010, 2016a); BAÑón \& SERRANo (2018)

Argyropelecus hemigymnus Cocco, 1829

Argyropelecus hemigymnus Cocco, 1829: DE Buen (1935a); Lozano Rey (1947); FARIÑa et al. (1985); SOLÓRZANO et al. (1988); RODRÍGUEZ Villanueva \& VÁzQuez (1995); Bañón et al. (2010, 2016a); BAÑón \& SerRano (2018)

Argyropelecus olfersii (Cuvier, 1829)

Argyropelecus olfersii (Cuvier, 1829): DE Buen (1935a); Lozano Rey (1947); Solórzano et al. (1988); PiÑEIRo et al. (1996); RodríGUEZ Villanueva \& VÁzquez (1995); Bañón et al. (2010, 2016a); BAÑón \& Serrano (2018)

Argyropelecus aculeatus Valenciennes, 1850

Argyropelecus aculeatus Valenciennes, 1850: BAÑón et al. (2010, 2016a); BAÑón \& SERrANo (2018)
Argyropelecus gigas Norman, 1930

Argyropelecus gigas Norman, 1930: BAÑóN et al. (2010, 2016a); Bañón \& SERrano (2018)

Maurolicus muelleri (Gmelin, 1789)

Maurolicus muelleri (Gmelin, 1789): DE Buen (1935a); Arté (1952a); Fernández et al. (1978); FARIÑA et al. (1985); SOLÓRZANO et al. (1988); RodRÍGUEZ VILlANUEVA \& VÁZQUEZ (1995); BAÑóN et al. (2010, 2016a); BAÑóN \& SERrano (2018)

Valenciennellus tripunctulatus (Esmark, 1871)

Valenciennellus tripunctulatus (Esmark, 1871): BAÑón et al. (2016a); BAÑóN \& SERRANO (2018)

Familia PHOSICHTHYIDAE Weitzman, 1974

Vinciguerria poweriae (Cocco, 1838): SolóRZANo et al. (1988); RODRÍGUEZ VILlANUEVA \& VÁzQuez (1995); BAÑón et al. (2010); BAÑóN \& Serrano (2018)

Ichthyococcus ovatus (Cocco, 1838)

Ichthyococcus ovatus (Cocco, 1838): BAÑóN et al. (2010); BAÑón \& SERRANo (2018)

Polymetme corythaeola (Alcock, 1898)

Polymetme corythaeola (Alcock, 1898): PIÑEIRO et al. (1996); BAÑón et al. (2010, 2016a); Bañón \& SERrano (2018)

Familia STOMIIDAE Bleeker, 1859

Rhadinesthes decimus (Zugmayer, 1911)

Astronesthes decimus Zugmayer, 1911: DE BuEN (1935a)

Rhadinesthes decimus (Zugmayer, 1911): Zugmayer en Lozano Rey (1947); SolórzaNo et al. (1988); Rodríguez Villanueva \& VÁzQuez (1995); BAÑón et al. (2010); BAÑóN \& Serrano (2018)

Stomias boa (Risso, 1810) (Fig. 2C)

Stomias boa boa (Risso, 1810): De BueN (1935a); Lozano Rey (1947); Franco \& Sola (1985); Solórzano et al. (1988); RodríGuez Villanueva \& VÁzQuez (1995); Bañón et al. (2010, 2016a); BAÑón \& Serrano (2018) 
Chauliodus sloani Bloch \& Schneider, 1801 (Fig. 2D)

Chauliodus sloani Bloch \& Schneider, 1801: PiñeIro et al. (1996); BAÑón et al. (2010, 2016a); BAÑón \& SERRANo (2018)

Photostomias guernei Collet, 1889 (Fig. 2E)

Photostomias guernei Collet, 1889: BAÑóN et al. (2010, 2016a); BAÑón \& SERRANo (2018)

Leptostomias gladiator (Zugmayer, 1911)

Leptostomias gladiator (Zugmayer, 1911): De Buen (1935a); Solórzano et al. (1988); Rodríguez Villanueva \& VÁzquez (1995); BAÑón et al. (2010); BAÑón \& SERRANo (2018)

Melanostomias gladiator (Zugmayer, 1911): Zugmayer en Lozano Rey (1947) [Melastomias gladiator]

Melanostomias bartonbeani Parr, 1927

Melanostomias bartonbeani Parr, 1927: BAÑón et al. (2010, 2016a); BAÑón \& SERRANO (2018)

Flagellostomias boureei (Zugmayer, 1913) (Fig. 2E)

Flagellostomias boureei (Zugmayer, 1913): BAÑón et al. (2016a); BAÑón \& SERrANo (2018)

Malacosteus niger Ayres, 1848 (Fig. 2F)

Malacosteus niger Ayres, 1848: BAÑón et al. (2016a); BAÑón \& SERrAno (2018)

Borostomias antarcticus (Lönnberg, 1905)

Borostomias antarcticus (Lönnberg, 1905): BAÑón et al. (2016a)

\section{Orden ATELEOPODIFORMES}

Familia ATELEOPODIDAE Bonaparte, 1850

Ijimaia loppei Roule, 1922

Ijimaia loppei Roule, 1922: Punzón \& Serrano (1998); Bañón (2002); Bañón et al. (2010); BAÑón \& SERrano (2018)

\section{Orden AULOPIFORMES \\ Familia CHLOROPHTHALMIDAE Gar- man, 1899}

Chlorophthalmus agassizi Bonaparte, 1840

Chlorophthalmus agassizi Bonaparte, 1840:

De Buen (1936); Solórzano et al. (1988);
Rodríguez Villanueva \& Vázquez (1995); PiÑEIRo et al. (1996); BAÑón et al. (2010); Bañón \& SERrano (2018)

\section{Familia IPNOPIDAE Gill, 1884}

Bathypterois dubius Vaillant, 1888

Bathypterois dubius Vaillant, 1888: PIÑEIRO et al. (1996); BAÑón et al. (2010; 2016a); BAÑóN \& Serrano (2018)

Familia PARALEPIDIDAE Bonaparte, 1835

Arctozenus risso (Bonaparte, 1840)

Paralepis rissoi (Bonaparte, 1840): DE Buen (1935a)

Notolepis rissoi (Bonaparte, 1840): SOLÓRZANo et al. (1988); RodRíguez VillanUeVA \& VÁzQUEZ (1995)

Arctozenus risso (Bonaparte, 1840): BAÑóN et al. (2010; 2016a); BAÑón \& SERRANO (2018)

Paralepis coregonoides Risso, 1820

Paralepis coregonoides Risso, 1820: DE Buen (1936); BAÑón et al. (2010); BAÑón \& SERrano (2018)

Magnisudis atlantica (Krøyer, 1868)

Magnisudis atlantica (Krøyer, 1868): BAÑóN et al. (2016a); BAÑón \& SerRano (2018)

Sudis hyalina Rafinesque, 1810

Sudis hyalina Rafinesque, 1810: ArTÉ (1952b); Solórzano et al. (1988); RodríGUEZ VillanueVa \& VÁzQuez (1995); BAÑón et al. (2010); BAÑón \& Serrano (2018)

Lestidiops sphyrenoides (Risso, 1820)

Paralepis sphyraenoides (Risso, 1826): DE BUEN (1936)

Familia BATHYSAURIDAE Fowler, 1944

Bathysaurus ferox Günther, 1878

Bathysaurus ferox Günther, 1878: BAÑóN et al. (2016a); BAÑón \& SERRANo (2018)

Familia ALEPISAURIDAE Swainson, 1839

Alepisaurus ferox Lowe, 1833

Alepisaurus ferox Lowe, 1833: ARTÉ (1952a,b); SOLÓRZANO et al. (1988); RODRÍGUEZ 
Villanueva \& VÁzquez (1995); Bañón et al. (2010); BAÑón \& SERrano (2018)

Familia ANOTOPTERIDAE Zugmayer 1911

Anotopterus pharao Zugmayer, 1911

Anotopterus pharao Zugmayer, 1911: DE Buen (1936)

\section{Orden MYCTOPHIFORMES}

Familia NEOSCOPELIDAE Jordan, 1901

Neoscopelus macrolepidotus Johnson, 1863

Neoscopelus macrolepidotus Johnson, 1863: PiñEIRO et al. (1996); BAÑóN (2002); BAÑón et al. (1997, 2010, 2016a); BAÑón \& SERRANO (2018)

Neoscopelus microchir Matsubara, 1943

Neoscopelus microchir Matsubara, 1943: BAÑÓN (2002); BAÑóN et al. (2002; 2010; 2016a); BAÑón \& Serrano (2018)

\section{Familia MYCTOPHIDAE Gill, 1893}

Myctophum punctatum Rafinesque, 1810

Myctophum punctatum Rafinesque, 1810: De Buen (1935a); Solórzano et al. (1988); Rodríguez Villanueva \& Vázquez (1995); BAÑóN et al. (2010, 2016a); BAÑóN \& SERRANO (2018)

Myctophum humboldti (Risso, 1810): DE BuEN (1936)

Diaphus dumerilii (Bleeker, 1856)

Diaphus dumerilii (Bleeker, 1856): BAÑóN et al. (2010); BAÑón \& SERrano (2018)

Diaphus luetkeni (Brauer 1904)

Diaphus luetkeni (Brauer 1904): De Buen (1936)

Electrona rissoi (Cocco, 1829)

Myctophum rissoi (Cocco, 1829): DE BUEN (1936)

Electrona rissoi (Cocco, 1829): BAÑón et al. (2016a); BAÑón \& SERrano (2018)

Ceratoscopelus maderensis (Lowe, 1839)

Lampanyctus maderensis (Lowe, 1839): DE Buen (1936); LozAno Rey (1947)

Ceratoscopelus maderensis (Lowe, 1839): Solórzano et al. (1988); Rodríguez VillaNUEVA \& VÁzQUEZ (1995); BAÑón et al. (2010, 2016a); BAÑón \& SerRano (2018)
Ceratoscopelus warmingii (Lütken, 1892)

Lampanyctus townsendi Eigenmann \& Eigenmann, 1889: LozANo REY (1947)

Ceratoscopelus warmingii (Lütken, 1892): Solórzano et al. (1988); Rodríguez VillaNUEVA \& VÁzQUEz (1995); BAÑón et al. (2010); BAÑón \& SERRANo (2018)

\section{Lampadena atlantica Maul, 1969}

Lampadena nitida Tåning, 1928: ArTÉ (1952a,b), ANAdón (1957)

Lampadena urophaos atlantica Maul, 1969: Solórzano et al. (1988); Rodríguez VillaNUEVA \& VÁzQUEZ (1995); BAÑóN et al. (2010); Bañón \& Serrano (2018)

Comentarios: Especie dudosa, citada originalmente para Galicia como L. nitida, sinónimo de Lampadena luminosa (Garman, 1899) (Worms, 2020), pero asignada posteriormente a Lampadena urophaos atlantica Maul, 1969 (SolórzANo et al., 1988), sinónimo de $L$. atlántica Maul, 1969.

Lampadena speculigera Goode \& Bean, 1896

Lampadena speculigera Goode \& Bean, 1896: BAÑón et al. (2010, 2016a); BAÑóN \& SERRANo (2018)

Lampanyctus crocodilus (Risso, 1810)

Lampanyctus gemmifer Goode \& Bean, 1896: De Buen (1935a)

Lampanyctus crocodilus (Risso, 1810): DE Buen (1936); Solórzano et al. (1988); RodríGuez Villanueva \& VÁzquez (1995); BañóN et al. (2010); Bañón \& Serrano (2018)

Comentarios: Estatus dudoso. De Buen (1935a) cita L. gemmifer para Galicia, que es considerado sinónimo de L. cocodrilus (Worms, 2020), que es el nombre asignado en las siguientes revisiones. Sin embargo, $L$. cocodrilus es citado también por DE BUEN (1935a) en otras regiones de España, pero no en Galicia, por lo que no hay criterios para saber a qué especie se refiere.

Lampanyctus alatus Goode \& Bean, 1896 Lampanyctus alatus Goode \& Bean, 1896: De Buen (1936)

Lampanyctus photonotus Parr, 1928

Lampanyctus taaningi Parr, 1929: De BueN en LozAno Rey (1947) 
Lampanyctus photonotus Parr, 1928: SolóRZANO et al. (1988); Rodríguez VILlanUEVA \& VÁzQuez (1995); BAÑón et al. (2010); BAÑóN \& Serrano (2018)

Lampanyctus intricarius Taning, 1928

Lampanyctus intricarius Taning, 1928: BAÑón et al. (2010); BAÑón \& SERRANo (2018)

Benthosema glaciale (Reinhardt, 1838)

Benthosema glaciale (Reinhardt, 1838): BAÑón et al. (2008, 2010); BAÑón \& SERRANo (2018)

Notoscopelus kroeyeri (Malm, 1861)

Notoscopelus elongatus (Costa, 1844): SoLÓRZANo et al. (1988); RodrígUEZ VILLANUEVA \& VÁzquez (1995)

Notoscopelus kroeyeri(Malm, 1861): BAÑóN et al. (2010, 2016a); BAÑón \& SERRANo (2018)

Lobianchia gemellarii (Cocco, 1838)

Diaphus gemellarii (Cocco, 1838): De Buen (1936)

\section{Orden LAMPRIFORMES}

Familia TRACHIPTERIDAE Swainson, 1839

A

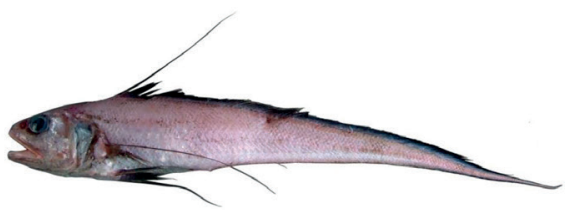

C

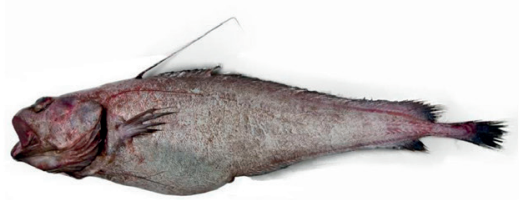

E

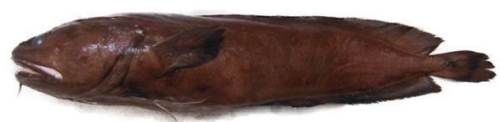

Trachipterus arcticus (Brünnich, 1788)

Trachipterus arcticus (Brünnich, 1788): ARTÉ (1952b); Solórzano et al. (1983, 1988); RodríGUEZ Villanueva \& VÁzQuez (1995); BaÑóN et al. (2007, 2010); BAÑón \& Serrano (2018)

Desmodema polystictum (Ogilby, 1898)

Desmodema polystictum (Ogilby, 1898): BAÑón et al. (2020)

Familia LAMPRIDAE Gill, 1862

Lampris guttatus (Brünnich, 1788)

Lampris guttatus (Brünnich, 1788): SolóRzano et al. (1983); Bañón \& Serrano (2018)

Comentarios: Solórzano et al. (1983) lo citan para Galicia con capturas accesorias en el palangre del marrajo, pero esta cita no se tiene en cuenta en las posteriores actualizaciones hasta que Bañón \& SERrano (2018) lo vuelven a incluir. Sabemos de al menos tres ejemplares capturados en aguas de Galicia por buques de arrastre y subastados en lonja en los años 2013, 2019 y 2020, que confirman su presencia en Galicia.

B

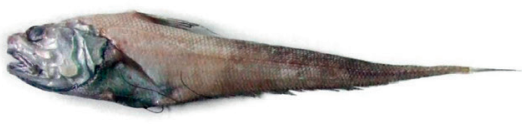

D

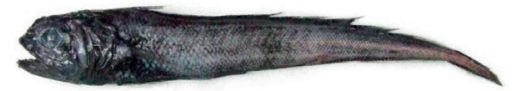

$\mathbf{F}$

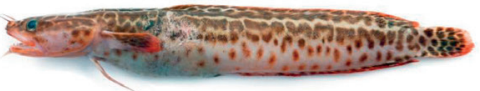

Figura 3. Peces Gadiformes presentes en Galicia. (A) Gadomus dispar (Vaillant, 1888) (Bathygadidae). (B) Coryphaenoides mediterraneus (Giglioli, 1893) (Macrouridae). (C) Lepidion guentheri (Giglioli, 1880) (Moridae). (D) Melanonus zugmayeri Norman, 1930 (Melanonidae). (E) Raniceps raninus (Linnaeus, 1758) (Gadidae). (F) Gaidropsarus granti (Regan, 1903) (Lotidae) Figure 3. Gadiform fishes in Galicia. (A) Gadomus dispar (Vaillant, 1888) (Bathygadidae). (B) Coryphaenoides mediterraneus (Giglioli, 1893) (Macrouridae). (C) Lepidion guentheri (Giglioli, 1880) (Moridae). (D) Melanonus zugmayeri Norman, 1930 (Melanonidae). (E) Raniceps raninus (Linnaeus, 1758) (Gadidae). (F) Gaidropsarus granti (Regan, 1903) (Lotidae) 


\section{Orden GADIFORMES}

Familia BATHYGADIDAE Jordan \& Evermann, 1898

Gadomus longifilis (Goode \& Bean, 1885)

Gadomus longifilis (Goode \& Bean, 1885): PiÑEIRo et al. (1996); BAÑóN (2002); BAÑóN et al. (2010, 2013, 2016a); Bañón \& SERrano (2018)

Gadomus dispar (Vaillant, 1888) (Fig. 3A) Gadomus dispar (Vaillant, 1888): BAÑón et al. (2013, 2016a); BAÑón \& Serrano (2018)

Bathygadus melanobranchus Vaillant, 1888

Bathygadus melanobranchus Vaillant, 1888: BAÑón et al. (2013, 2016a); BAÑón \& SERRANO (2018)

Familia MACROURIDAE Bonaparte, 1831

Trachyrincus scabrus (Rafinesque, 1810)

Macrurus trachyrhynchus (Risso, 1810): De Buen (1919)

Trachyrhynchus trachyrhynchus (Risso, 1810): De Buen (1935a); Fernández et al. (1978); SOLÓRZANO et al. (1983, 1988); RodRÍGuez Villanueva \& VÁzQuez (1995); Piñeiro et al. (1996)

Trachyrincus scabrus (Rafinesque, 1810): BAÑón et al. (2010, 2016a); BAÑóN \& SERRANO (2018)

Hymenocephalus italicus Giglioli, 1884

Hymenocephalus italicus Giglioli, 1884: PIÑEIRO et al. (1996); BAÑóN (2002); BAÑóN et al. (2010, 2016a); BAÑón \& SERrano (2018)

Coelorinchus labiatus (Koelher, 1896)

Coelorinchus vaillanti Roule, 1916: ARTÉ (1952a)

Coelorinchus occa (Goode \& Bean, 1885): SOlÓRzAno et al. (1988); Rodríguez VillanUEVA \& VÁzQuez (1995)

Coelorinchus labiatus (Koelher, 1896): BAÑón et al. (2010, 2016a); BAÑón \& SERRANO (2018)

Coelorinchus caelorhincus (Risso, 1810)

Coelorhynchus coelorhynchus (Risso, 1810): SOLÓRZANo et al. (1983)
Coelorinchus caelorhincus (Risso, 1810): BAÑón et al. (2010, 2016a) [Coelorinchus caelorinchus]; BAÑón \& SERRANo (2018)

Coryphaenoides rupestris Gunnerus, 1765

Coryphaenoides rupestris Gunnerus, 1765: Solórzano et al. (1983); PiñeIro et al. (1996); BAÑóN (2002); BAÑón et al. (2010, 2016a); Bañón \& SERrano (2018)

Coryphaenoides mediterraneus (Giglioli, 1893) (Fig. 3B)

Coryphaenoides mediterraneus (Giglioli, 1893): BAÑón et al. (2016a); BAÑóN \& SERRANO (2018)

Coryphaenoides guentheri (Vaillant, 1888)

Coryphaenoides guentheri (Vaillant, 1888): BAÑón et al. (2016a); BAÑón \& SERRANO (2018)

Cetonurus globiceps (Vaillant, 1884)

Cetonurus globiceps (Vaillant, 1884): PIÑEIRo et al. (1996); BAÑóN (2002); BAÑóN et al. (2010); Bañón \& Serrano (2018)

Malacocephalus laevis (Lowe, 1843)

Malacocephalus laevis (Lowe, 1843): DE BUEN (1919, 1935a); Fernández et al. (1978, 1981); SOlÓRZANO et al. (1983, 1988); RodRÍGUEZ Villanueva \& VÁzQuez (1995); PiñeIRo et al. (1996); BAÑóN et al. (2010, 2016a); BAÑÓN \& Serrano (2018)

Nezumia aequalis (Günther, 1878)

Macrourus serratus Lowe, 1843: DE BUEN (1935a)

Nezumia aequalis (Günther, 1878): SolóRZANo et al. (1983, 1988); RodrígUEZ VILlANUEVA \& VÁzQUEZ (1995); BAÑóN et al. (2010, 2016a); BAÑón \& Serrano (2018)

Nezumia sclerorhynchus (Valenciennes, 1838)

Solórzano et al. (1988); Rodríguez VillaNueVa \& VÁzquez (1995); PiñeIro et al. (1996); BAÑón et al. (2010); BAÑón \& SERRANo (2018)

Familia MORIDAE Moreau, 1881

Antimora rostrata (Günther, 1878)

Antimora rostrata (Günther, 1878): DE BUEN (1935a); SolórzANo et al. (1988); RodríGUEz 
Villanueva \& VÁzQuez (1995); Bañón et al. (2010, 2016a); BAÑón \& SERRANo (2018)

Gadella maraldi (Risso, 1810)

Gadella maraldi (Risso, 1810): BAÑón et al. (2010); BAÑón \& SERRANo (2018)

Halargyreus johnsonii Günther, 1862

Halargyreus johnsonii Günther, 1862: PiñeIro et al. (1996); BAÑón (2002); BAÑón et al. (2010, 2016a); Bañón \& Serrano (2018); De CARLos et al. (2020)

Physiculus dalwigki Kaup, 1858

Physiculus dalwigki Kaup, 1858: BAÑóN (2002); BAÑón et al. (2002, 2010, 2016a); BAÑón \& SERRANo (2018)

Mora moro (Risso, 1810)

Mora moro (Risso, 1810): PIÑEIRo et al. (1996); BAÑón (2002); BAÑón et al. (2010, 2016a); BAÑón \& SERRANo (2018)

\section{Lepidion lepidion (Risso, 1810)}

Lepidion eques (Günther, 1887): SolóRZANO et al. (1983, 1988); Rodríguez VillanueVa \& VÁzQUez (1995); PIÑEIRo et al. (1996); BAÑóN et al. (2010), Lepidion lepidion Risso, 1810: BAÑón et al. (2013, 2014, 2016a); BAÑóN \& Serrano (2018); Barros García et al. (2016b, 2020)

Comentarios: Especie muy estudiada a nivel taxonómico en Galicia, probando que la especie que habita el Atlántico y el Mediterráneo es la misma, contrariamente a lo que se creía hasta ese momento. (BAÑón et al., 2013; Barros García et al., 2016b, 2020).

Lepidion guentheri (Giglioli, 1880) (Fig. 3C)

Lepidion guentheri (Giglioli, 1880): BAÑóN et al. (2016a); BAÑón \& SERrano (2018)

Guttigadus latifrons (Holt \& Byrne 1908) Guttigadus latifrons (Holt \& Byrne 1908): BAÑón et al. (2016a); BAÑón \& SERRANo (2018)

Familia MELANONIDAE Goode \& Bean, 1896

Melanonus zugmayeri Norman, 1930 (Fig. 3D)

Melanonus zugmayeri Norman, 1930: PIÑEIRO et al. (1996); BAÑóN (2002); BAÑÓN et al. (2010, 2016a); BAÑón \& SERRANo (2018)
Familia GADIDAE Rafinesque, 1810

Raniceps raninus (Linnaeus, 1758) (Fig. 3E)

Raniceps raninus (Linnaeus, 1758): DE BUEN (1919, 1935a); LozANO REy (1919); SolóRZANO et al. (1983, 1988); BAÑón et al. (2010); BAÑóN \& Serrano (2018)

Trisopterus luscus (Linnaeus, 1758)

Gadus barbatus Linnaeus, 1758: CORNIDE (1788)

Gadus luscus Linnaeus, 1758; De Buen (1919, 1935a); Lozano Rey (1919); Fernández et al. (1978, 1981); FARIÑa et al. (1985); Solórzano et al. (1983, 1988); RodríGuez Villanueva \& VÁzQuez (1995); PiÑeIro et al. (1996); BAÑóN et al. (2010); BAÑón \& SERRANo (2018).

Trisopterus minutus (Linnaeus, 1758)

Trisopterus minutus (Linnaeus, 1758): STEINDACHNER en Lozano Rey (1960); FernáNDEZ et al. (1978, 1981); FARIÑA et al. (1985); Solórzano et al. (1983, 1988); Rodríguez Villanueva \& VÁzQuez (1995); BAÑón et al. (2010); BAÑón \& SERRANo (2018)

Trisopterus capelanus (Lacepède, 1800)

Trisopterus capelanus (Lacepède, 1800): GAEMers (2016)

Comentarios: Registro dudoso. GaEmers (2016) cita esta especie en la Ría de Arousa, basándose en otolitos de sedimentos recientes y del Holoceno recogidos por CADÉE (1968). Sin embargo, esta especie es considerada por algunos autores endémica del Mediterráneo y, salvo esta excepción, no ha sido nunca citada en el Atlántico.

Gadus morhua Linnaeus, 1758

Gadus morhua Linnaeus, 1758: López SEOANE (1866) [Gadus morrhua]; BAÑón et al. (2010); Bañón \& Serrano (2018)

Comentarios: Especie boreal que tiene en Galicia y Portugal su límite sur de distribución, con apariciones esporádicas de ejemplares errantes.

Micromesistius poutassou (Risso, 1827)

Gadus poutassou (Risso, 1827): CligNY (1905); FERnÁndeZ et al. (1978, 1981); FARIÑA 
et al. (1985); SolóRzANo et al. (1983, 1988); Rodríguez Villanueva \& VÁzquez (1995); BAÑón et al. (2010); BAÑón \& SERRANo (2018)

Gadiculus argenteus Guichenot, 1850

Gadiculus argenteus Guichenot, 1850: De Buen (1935a); Fernández et al. (1978, 1981); FARIÑA et al. (1985); SolóRzANo et al. (1983, 1988); Rodríguez Villanueva \& VÁzQUEZ (1995); BAÑóN et al. (2010); BAÑóN $\&$ Serrano (2018)

Merlangius merlangus (Linnaeus, 1758)

Gadus merlangus Linnaeus, 1758: CORNIDE (1788); De Buen (1935a)

Merlangus vulgaris Fleming, 1828: LÓPEZ SEOANE (1866)

Merlangius merlangus (Linnaeus, 1758): Solórzano et al. (1983, 1988); RodríGUEZ Villanueva \& VÁzQuez (1995); Bañón et al. (2010); BAÑón \& SERRANo (2018)

Pollachius pollachius (Linnaeus, 1758)

Gadus pollachius Linnaeus, 1758: CORNIDE (1788); De Buen (1935a)

Pollachius typus Bonaparte, 1846: PAz Graells (1870)

Merlangus pollachius (Linnaeus, 1758): DE BuEN (1919)

Pollachius pollachius (Linnaeus, 1758): FERnÁNdez et al. (1978, 1981); SOlóRzANO et al. (1983, 1988); Rodríguez Villanueva \& VÁzQuez (1995); BAÑón et al. (2010); BAÑóN \& SerRano (2018)

\section{Familia LOTIDAE Bonaparte, 1835}

Gaidropsarus macrophthalmus (Günther, 1867) Gaidropsarus biscayensis (Collett, 1890): De Buen (1935a)

Gaidropsarus macrophthalmus (Günther, 1867): Solórzano et al. (1988); RodríGUez Villanueva \& Vázquez (1995); Piñeiro et al. (1996); BAÑón et al. (2010); BARRos GARCÍA et al. (2018); BAÑón \& SERRANO (2018)

Gaidropsarus granti (Regan, 1903) (Fig. 3F) Gaidropsarus granti (Regan, 1903): BAÑóN et al. (2002, 2010, 2016a); BARros García et al. (2018); BAÑón \& Serrano (2018)
Gaidropsarus vulgaris (Cloquet, 1824)

Motella tricirrata Nilsson, 1832: PAZ Graells (1870)

Onos tricirratus (non Brünnich, 1768): DE BuEN (1919) [Onos tricirrata Bl]

Gadus tricirratus Brünnich, 1768: LozANo REy (1919, 1960)

Gaidropsarus vulgaris (Cloquet, 1824): FERNÁNDEZ et al. (1978, 1981); FARIÑA (1985); Solórzano et al. (1983, 1988); Rodríguez Villanueva \& Vázquez (1995); Bañón et al. (2010); Barros García et al. (2018); BAÑóN \& Serrano (2018)

Comentarios: Aunque G. tricirratus (Brünnich) es considerado un sinónimo de G. mediterraneus (WoRMs, 2020), en este caso LozANo REY (1919) se refiere a esta especie como Onos fusca (Risso, 1810), por lo que G. tricirratus nombra con toda probabilidad a $G$. vulgaris.

Gaidropsarus mediterraneus (Linnaeus, 1758)

Gadus mediterraneus Linnaeus, 1758: CorNIDE (1788)

Onos fusca (Risso, 1810): Lozano ReY (1919)

Gaidropsarus guttatus (Collet, 1890): FERNÁNDEZ et al. (1978)

Gaidropsarus mediterraneus (Linnaeus, 1758): De Buen (1935a); Lozano Rey (1960); FERnÁndez et al. (1981); FARIÑa (1985); Solórzano et al. (1983, 1988); RodríGUEZ Villanueva \& VÁzQuez (1995); Bañón et al. (2010); BARros García et al. (2018); BAÑóN \& Serrano (2018)

Comentarios: Fernández et al. (1978) citan por vez primera G. guttattus en la Europa continental, sin embargo, esta especie es considerada actualmente como sinónimo de G. mediterraneus (BARros GARCÍA et al. 2018).

Ciliata mustela (Linnaeus, 1758)

Onos mustela (Linnaeus, 1758): DE BUEN (1919)

Gaidropsarus mustela (Linnaeus, 1758): De Buen (1935a) [Gaidropsarus mustella]; De Buen (1960)

Ciliata mustela (Linnaeus, 1758): FARIÑA (1985); SOlÓRZANO et al. (1983, 1988); RodRÍguez Villanueva \& Vázquez (1995); Bañón et al. (2010); Bañón \& Serrano (2018) 
Molva molva (Linnaeus, 1758)

Molva molva (Linnaeus, 1758): FERnÁNDEZ et al. (1981); Solórzano et al. (1983, 1988); Rodríguez Villanueva \& VÁzQuez (1995); BAÑón et al. (2010); BAÑón \& SERRANo (2018)

Molva macrophthalma (Rafinesque, 1810)

Molva byrkelange macrophthalma (Rafinesque, 1810): LozAno Rey (1919)

Molva elongata (Risso, 1810): De Buen (1935a)

Molva dypterygia macrophthalma (Rafinesque, 1810): Fernández et al. (1978, 1981); FARIÑA (1985); SOlÓRZANO et al. (1988)

Molva dypterygia (Pennant, 1784): SolóRZANo et al. (1983); Rodríguez VillanUeVA \& VÁzQuez (1995); PiñeIro et al. (1996)

Molva macrophthalma (Rafinesque, 1810): BAÑón et al. (2010); BAÑón \& SERrano (2018)

Comentarios: Si bien las antiguas denominaciones en Galicia de $M$. dypterygia (Pennant) son atribuibles a $M$. macrophthalma (Rafinesque), M. dypterigia es actualmente una especie válida y Galicia está dentro de su área de distribución, por lo que su presencia en nuestras aguas es probable.

Familia PHYCIDAE Swainson, 1838

Phycis phycis (Linnaeus, 1766)

Urophycis phycis (Linnaeus, 1758): LozANO Rey (1919); De Buen (1935a)

Phycis phycis (Linnaeus, 1766): De Buen (1919); Lozano Rey (1960); Solórzano et al. (1983, 1988); Rodríguez Villanueva \& VÁzquez (1995); PiñeIro et al. (1996); BAÑóN et al. (2010); BAÑón \& SERrano (2018)

Phycis blennoides (Brünnich, 1768)

Phycis blennoides (Brünnich, 1768): LÓPEZ SeOANE (1866) [Phycis blenoides]; PAz Graells (1870) [Phycis blenoides]; FERnÁNDEZ et al. (1978, 1981); FAriña (1985); Solórzano et al. (1983, 1988); Rodríguez Villanueva \& VÁzQuez (1995); PiñeIro et al. (1996); BAÑóN et al. (2010, 2016a); BAÑón \& SERrANo (2018)

Urophycis blennioides (Brünnich, 1768): DE BUEN (1935a)

Familia MERLUCCIDAE Rafinesque, 1815

Merluccius merluccius (Linnaeus, 1758)

Gadus merluccius Linnaeus, 1758: CORNIDE (1788)
Merluccius vulgaris Fleming, 1828: LÓPEZ SeOANe (1866)

Merlucius esculentus Risso, 1827: PAZ Graells (1870)

Molva byrkelange(Walbaum, 1792): CLIGNY (1903)

Merluccius merluccius (Linnaeus, 1758): De Buen (1919, 1935a); Lozano Rey (1919); FERNÁNDEZ et al. (1978, 1981); FARIÑA et al. (1985); SOlÓRZANO et al. (1983, 1988); RodRÍGUEZ Villanueva \& VÁzQuez (1995); PiñeIRo et al. (1996); BAÑón et al. (2010, 2016a); BAÑÓN \& Serrano (2018).

Comentarios: Especie ampliamente citada en Galicia, capturada desde la antigüedad por su alto valor comercial. CoRnIDE (1788) la cita por vez primera siguiendo a LINNAEUS (1758) pero en vez de citar el binomio de Linneo Gadus merluccius, lo que hace es citar su descripción "Gadus dipterygius imberbis, maxilla inferiore longiore".

\section{Orden OPHIDIIFORMES}

Familia OPHIDIIDAE Rafinesque, 1810

Ophidion barbatum Linnaeus, 1758

Ophidion barbatum Linnaeus, 1758: CoRNide (1788); López SeoAne (1866); De Buen (1935b); SOlÓRZANo et al. (1983, 1988); RodRÍGuez Villanueva \& VÁzQuez (1995); BañóN et al. (2010); BAÑón \& Serrano (2018)

Spectrunculus grandis (Günther, 1877)

Spectrunculus grandis (Günther, 1877): BAÑón et al. (2016a); BAÑón \& SERRANO (2018)

Familia CARAPIDAE Poey, 1867

Carapus acus (Brünnich, 1768)

Carapus acus (Brünnich, 1768): FERNÁNDEZ et al. (1978); FARIÑA et al. (1985); SOlóRZANO et al. (1988); Rodríguez Villanueva \& VÁzQUEZ (1995); BAÑóN et al. (2010); BAÑóN \& SERrano (2018)

Echiodon drummondii Thompson, 1837

Echiodon drummondii Thompson, 1837: Solórzano et al. (1988); Rodríguez VillaNUEVA \& VÁzQUEZ (1995); BAÑón et al. (2010); BAÑón \& SERRANO (2018) 
Echiodon dentatus (Cuvier, 1829)

Echiodon dentatus (Cuvier, 1829): FERNÁNDEZ et al. (1978); FARIÑA et al. (1985); Solórzano et al. (1983, 1988); RodríGUEZ Villanueva \& VÁzQUez (1995); BAÑón et al. (2010); BAÑón \& SERRANo (2018)

Familia BYTHITIDAE Gill, 1861

Sciadonus cryptophthalmus (Zugmayer, 1911)

Leucochlamys cryptophthalmus Zugmayer, 1911: De Buen (1935b)

Sciadonus cryptophthalmus (Zugmayer, 1911): Solórzano et al. (1988); RodríGUez Villanueva \& VÁzQUez (1994); BAÑón et al. (2010); BAÑón \& SERRANO (2018)

Cataetyx alleni (Byrne, 1906)

Cataetyx alleni (Byrne, 1906): BAÑón et al. (2016a); BAÑón \& SERRANO (2018)

Cataetyx laticeps Koefoed, 1927

Cataetyx laticeps Koefoed, 1927: BAÑón et al. (2016a); Bañón \& Serrano (2018)

\section{Orden BATRACHOIDIFORMES} Familia BATRACHOIDIDAE Jordan, 1896 (1835)

Halobatrachus didactylus (Bloch \& Schneider, 1801)

Halobatrachus didactylus (Bloch \& Schneider, 1801): BAÑón et al. (2019); BAÑóN et al. (2020)

\section{Orden LOPHIIFORMES}

Familia LOPHIIDAE Rafinesque, 1810

\section{Lophius budegassa Spinola, 1807}

Lophius budegassa Spinola, 1807: LozANO Rey (1919); De Buen (1935b); Fernández etal. (1978, 1981); FARIÑA et al. (1985); SOLÓRZANO et al. (1983, 1988); RodríGUEZ VillanUeVA \& VÁzQuez (1995); PiñeIro et al. (1996); BAÑóN \& Armesto (2003); BAÑón et al. (2010); BAÑón \& SerRano (2018)

Lophius piscatorius Linnaeus, 1758

Lophius piscatorius Linnaeus, 1758: CoRNide (1788); López SeoAne (1866); De Buen
(1919, 1935b); Lozano Rey (1919); FernánDEZ et al. (1978, 1981); FARIÑA et al. (1985); Solórzano et al. (1983, 1988); RodríGuez Villanueva \& VÁzQuez (1995); Piñeiro et al. (1996); BAÑón et al. (2010, 2016a); BAÑón \& SERrano (2018)

\section{Familia CHAUNACIDAE Gill, 1863}

Chaunax pictus Lowe, 1846

Chaunax pictus Lowe, 1846: PIÑEIRo et al. (1996); BAÑón et al. (1997, 2010, 2016a); BAÑón \& SERRANo (2018)

Familia CERATIIDAE Gill, 1861

Ceratias holboelli Krøyer, 1845

Ceratias holboelli Krøyer, 1845: BAÑón et al. (2010); BAÑón \& SERRANo (2018)

Familia LINOPHRYNIDAE Regan, 1925

\section{Linophryne coronata Parr, 1927}

Linophryne coronata Parr, 1927: BAÑón et al. (2006); BAÑóN et al. (2010, 2016a); BAÑóN \& Serrano (2018)

\section{Familia HIMANTOLOPHIDAE Gill, 1861}

Himantolophus groenlandicus Reinhardt, 1837

Himantolophus groenlandicus Reinhardt, 1837: Bañón \& Serrano (2018)

\section{Orden GOBIESOCIFORMES}

Familia GOBIESOCIDAE Bleeker, 1859

Lepadogaster lepadogaster (Bonnaterre, 1788)

Lepadogaster gouanii Risso, 1810: LÓPEZ SEOANE (1866) [Lepadogaster gouan]; LozAno Rey (1919); De Buen (1935b) [Lepadogaster gouani Lacépède 1798]

Lepadogaster lepadogaster (Bonnaterre, 1788): Lozano Rey (1960); Solórzano et al. (1983, 1988); Rodríguez Villanueva \& VÁzQUEz (1995); BAÑón et al. (2010); BAÑóN \& Serrano (2018)

Lepadogaster purpurea (Bonnaterre, 1788)

Lepadogaster purpurea (Bonnaterre, 1788): Henriques et al. (2002); BAÑón et al. (2010); Bañón \& SERrano (2018) 
Lepadogaster candolii Risso, 1810

Lepadogaster candollei Risso, 1810: SolóRZANO et al. (1983)

Lepadogaster candolii Risso, 1810: BAÑóN et al. (2010); BAÑón \& SerRano (2018)

Diplecogaster bimaculata (Bonnaterre, 1788)

Mirbelia bimaculata (Pennant, 1776): DE Buen (1935b)

Lepadogaster bimaculatus (Bonnaterre, 1788): STeindachner en Lozano Rey (1960)

Diplecogaster bimaculata (Bonnaterre, 1788): Solórzano et al. (1983, 1988); FARIÑa et al. (1985); Rodríguez Villanueva \& VÁzQuez (1995); BAÑón et al. (2010); BAÑóN \& Serrano (2018)

Apletodon dentatus dentatus (Facciolà, 1887)

Apletodon microcephalus microcephalus (Brook, 1890): SolórzANo et al. (1983, 1988)

Apletodon dentatus dentatus (Facciolà, 1887): Iglesias (1981); RodríGUEZ VILlaNUEVA \& VÁzQuEz (1995); BAÑóN et al. (2010); BAÑóN \& SERRANo (2018)

\section{Orden ATHERINIFORMES}

Familia ATHERINIDAE Risso, 1827

Atherina presbyter Cuvier, 1829

Atherina hepsetus Linnaeus, 1758: CORNIDE (1788) [Atherina epsetus]; López SEOAne (1866) [Atherina hepsatus];

Atherina presbyter Cuvier, 1829: PAz Graells (1870); De Buen (1935b); Lozano REY (1947); FERNÁNDEZ et al. (1978); SOLÓRZANo et al. (1983, 1988); Rodríguez VILlanUEVA \& VÁzQUEz (1995); BAÑóN et al. (2010); BAÑóN \& Serrano (2018)

Comentarios: Aunque $A$. hepsetus L. es considerada una especie válida distribuida hasta Galicia, las antiguas denominaciones con este nombre se corresponden con mayor probabilidad a $A$. presbyter.

Atherina boyeri Risso, 1810

Atherina boyeri Risso, 1810: SOLÓRzANO et al. (1983)

Comentarios. Registro dudoso. Citada únicamente por SolóRzANo et al. (1983) como escasa y de distribución local. No ha vuelto a ser citada en las revisiones posteriores.

\section{Orden BELONIFORMES}

Familia SCOMBERESOCIDAE Bleeker, 1859

Scomberesox saurus (Walbaum, 1792)

Exos osseus Linnaeus, 1758: CoRNIDE (1788)- error de identificación

Scomberesox saurus (Walbaum, 1792): DE Buen (1936); Solórzano et al. (1983, 1988); Rodríguez Villanueva \& VÁzQuez (1995); BAÑón et al. (2010); BAÑón \& SERrano (2018)

Familia BELONIDAE Bonaparte, 1835

Belone belone (Linnaeus, 1761)

Exos bellone Linnaeus, 1758: CORNIDE (1788) [Exos belone]

Belone rostrata Faber, 1829: López SEOANE (1866); Paz Graells (1870)

Rhamphistoma belone (Linnaeus, 1758): Lozano Rey (1919); De Buen (1935a)

Belone belone (Linnaeus, 1761): De BUEN (1919); Lozano Rey (1947); Solórzano et al. (1983, 1988); RodríGuez Villanueva \& VÁzQUEZ (1995); BAÑón et al. (2010); BAÑóN \& Serrano (2018)

Comentarios: CORNIDE (1788) describe dos especies de agujas sin apenas diferencias taxonómicas, pero cita que la aguja paladar $E$. belone alcanza tallas mayores que la aguja $E$. osseus, que es un carácter que tiene $B$. belone respecto a $S$. saurus.

Familia EXOCOETIDAE Risso, 1827

Cheilopogon heterurus (Rafinesque, 1810)

Cheilopogon heterurus (Rafinesque, 1810): SOlÓRZANO et al. (1983, 1988); RodRÍGUEZ Villanueva \& VÁzQuez (1995); Bañón et al. (2008a, 2010); BAÑón \& Serrano (2018)

Cheilopogon pinnatibarbatus pinnatibarbatus (Bennett, 1831)

Cypselurus lineatus (Valenciennes, 1847): De Buen (1936)

\section{Orden ESTEPHANOBERYCIFORMES}

Familia MELAMPHAIDAE Gill 1893

Melamphaes microps (Günther, 1878)

Melamphaes mizolepis (Günther, 1878): DE Buen (1935a); Lozano Rey (1952)

Melamphaes microps (Günther, 1878): SolóRzAno et al. (1988); Rodríguez VILlanUEVA 
\& VÁzquez (1995); BAÑón et al. (2008a, 2010); BAÑón \& SERRANo (2018)

Comentarios: Identificación dudosa. Solórzano et al. (1988) identifican M. mizolepis como M. microps, pero actualmente M. mizolepis es considerado un sinónimo de Scopelogadus mizolepis (Günther, 1878). Ambas especies son similares morfológicamente y Galicia está dentro de su área de distribución.

A

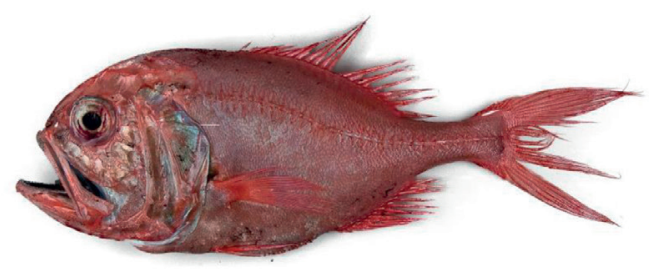

C

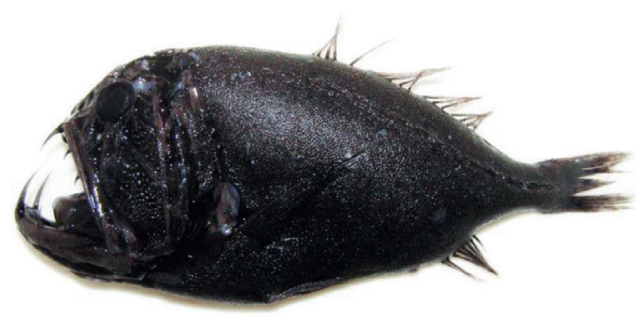

Familia DIRETMIDAE Gill, 1893

Diretmichthys parini (Post \& Quéro, 1981) (Fig. 4B)

Diretmichthys parini (Post \& Quéro, 1981): BAÑón et al. $(2010,2016 a)$; BAÑón \& SERRANO (2018)

Diretmus argenteus Johnson, 1864

Diretmus argenteus Johnson, 1864: De BuEN

B

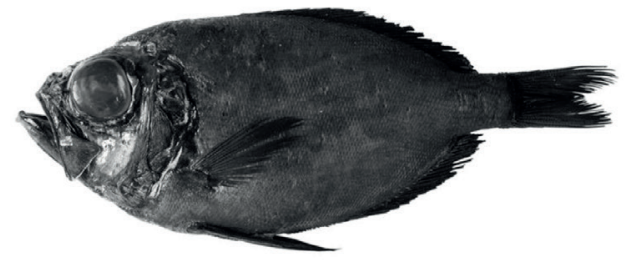

D

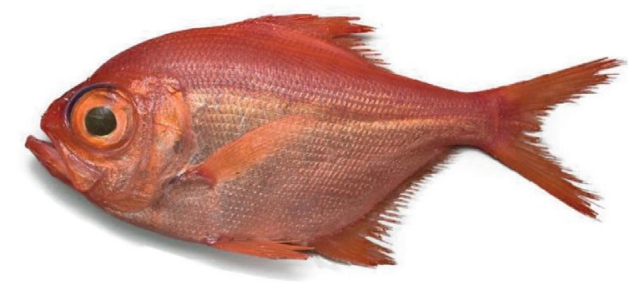

Figure 4. Peces Beryciformes presentes en Galicia. (A) Hoplostethus atlanticus Collett, 1889 (Trachichthyidae). (B) Diretmichthys parini (Post \& Quéro, 1981) (Diretmidae). (C) Anoplogaster cornuta (Valenciennes, 1833) (Anoplogasteridae) (D) Beryx decadactylus Cuvier, 1829 (Berycidae).

Figure 4. Beryciform fishes in Galicia (A) Hoplostethus atlanticus Collett, 1889 (Trachichthyidae). (B) Diretmichthys parini (Post \& Quéro, 1981) (Diretmidae). (C) Anoplogaster cornuta (Valenciennes, 1833) (Anoplogasteridae) (D) Beryx decadactylus Cuvier, 1829 (Berycidae).

\section{Orden BERYCIFORMES}

Familia TRACHICHTHYIDAE Bleeker, 1856

Hoplostethus mediterraneus Cuvier, 1829

Hoplostethus mediterraneus Cuvier, 1829: Cligny 1905; De Buen (1936); Solórzano et al. (1983, 1988); Rodríguez Villanueva \& VÁzquez (1995); PiñeIro et al. (1996); BAÑóN et al. (2010, 2016a); BAÑón \& SERRANo (2018)

Hoplostethus atlanticus Collett, 1889 (Fig. 4A)

Hoplostethus atlanticus Collett, 1889: PiñeIro et al. (1996); BAÑón et al. (2010, 2016a); BAÑón \& SERRANo (2018)

Hoplostethus cadenati Quéro, 1974

Hoplostethus cadenati Quéro, 1974: PIÑEIRO et al. (1996); BAÑón et al. (1997, 2010, 2016a); BAÑón \& SERRANo (2018) (1935a); Lozano Rey (1952); Piñeiro et al. (1996); BAÑón et al. (2010, 2016a); BAÑóN \& Serrano (2018)

Familia ANOPLOGASTRIDAE Gill, 1893

Anoplogaster cornuta (Valenciennes, 1833) (Fig. 4C)

Anoplogaster cornuta (Valenciennes, 1833): PiñeIro et al. (1996); BAÑóN (2002); BAÑón et al. (2010); BAÑón \& SERRANo (2018)

Familia BERYCIDAE Lowe, 1839

Beryx splendens Lowe, 1834

Beryx splendens Lowe, 1834: ClignY (1903); De Buen (1919, 1935a); Lozano Rey (1952); Solórzano et al. (1988); Rodríguez Villanueva \& Vázquez (1995); Piñeiro et al. 
(1996); BAÑón et al. (2010, 2016a); BAÑón \& SERrano (2018)

Beryx decadactylus Cuvier, 1829 (Fig. 4D)

Beryx decadactylus Cuvier, 1829: CLIGNY (1903); De Buen (1919, 1935a); Lozano Rey (1952); FERNÁNDEZ et al. (1978); FARIÑA et al. (1985); SolórZANo et al. (1983, 1988); RodríGuez VillanueVa \& VÁzQuez (1995); PiñeIro et al. (1996); BAÑóN et al. (2010, 2016a); BAÑóN \& Serrano (2018)

\section{Orden ZEIFORMES}

Familia PARAZENIDAE McAllister, 1968

Cyttopsis rosea (Lowe, 1843)

Cyttus roseus (Lowe, 1843): Fernández et al. (1978)

Cyttopsis roseus (Lowe, 1843): SOlÓRzANO et al. (1983, 1988); Rodríguez Villanueva \& VÁzQuez (1995); PiñeIro et al. (1996); BAÑóN et al. (1997)

Cyttopsis rosea (Lowe, 1843): BAÑón et al. (2010); BAÑón \& SERRANo (2018)

Familia ZEIDAE Rafinesque, 1815

Zenopsis conchifer (Lowe, 1852)

Zenopsis conchifera (Lowe, 1852): BAÑóN et al. (2010)

Zenopsis conchifer (Lowe, 1852): QuÉRO (1972); SOLÓRZANO et al. (1983, 1988); RodRÍGUez Villanueva \& VÁzQuez (1995); Bañón \& Serrano (2018)

Comentarios: El primer ejemplar citado en Galicia, de $56 \mathrm{~cm}$ LT, fue capturado el 24 de Junio 1970 entre Vigo y Finisterre, a 280 m de profundidad, por el barco francés "Dadas" (QUÉro, 1972)

Zeus faber Linnaeus, 1758

Zeus faber Linnaeus, 1758: CoR NIDE (1788); López Seoane (1866); Paz Graells (1870); De Buen (1919, 1935a); Lozano Rey (1919, 1952); FERNÁNDEZ et al. (1978); FARIÑA et al. (1985); Solórzano et al. (1983, 1988); Rodríguez VillanueVa \& VÁzQUez (1995); BAÑón et al. (2010); BAÑón \& SERRANo (2018)

Familia ZENIONTIDAE Myers, 1960 Zenion hololepis (Goode \& Bean, 1896) Zenion hololepis (Goode \& Bean, 1896): BAÑón \& SERRANo (2018)
Familia OREOSOMATIDAE Bleeker, 1859

Neocyttus helgae (Holt \& Byrne, 1908): FARIÑA et al. (1999); BAÑóN et al. (2010, 2016a); BAÑón \& SERRANO (2018)

Familia GRAMMICOLEPIDIDAE Poey, 1873

Grammicolepis brachiusculus Poey, 1873

Grammicolepis brachiusculus Poey, 1873: Quéro (1970, 1979); Quéro \& Robles (1977), BAÑón \& SERRANo (2018)

Comentarios: La primera cita para Galicia fue publicada en Francia, por lo que ha pasado desapercibida durante largo tiempo. Un ejemplar de $535 \mathrm{~mm}$ LT fue capturado el 3 Octubre de 1966, cerca de las islas Sisargas, en $43^{\circ} 50^{\prime} \mathrm{N}-8^{\circ} 40^{\prime} \mathrm{W}$, a $600 \mathrm{~m}$ de profundidad (QUÉRO, 1970).

\section{Orden GASTEROSTEIFORMES}

Familia GASTEROSTEIDAE Bonaparte, 1831

Gasterosteus aculeatus Linnaeus, 1758

Gasterosteus aculeatus Linnaeus, 1758: BAÑón et al. (2010); BAÑón \& SERRANo (2018)

Spinachia spinachia (Linnaeus, 1758)

Spinachia spinachia (Linnaeus, 1758): DE Buen (1919, 1935a); Lozano Rey (1947); FARIÑA et al. (1985); SolórzANo et al. (1983, 1988); Rodríguez Villanueva \& VázQuez (1995); Fernández Cordeiro (1997); BAÑón et al. (2010); BAÑón \& SerRano (2018)

\section{Orden SYNGNATHIFORMES}

Familia FISTULARIIDAE Stark, 1828

Fistularia petimba Lacepède, 1803

Fistularia petimba Lacepède, 1803: BAÑóN \& SANDE (2008); BAÑón et al. (2010); BAÑón \& Serrano (2018)

Familia DACTYLOPTERIDAE Gill, 1861

Dactylopterus volitans (Linnaeus, 1758)

Dactylopterus volitans (Linnaeus, 1758): BAÑón et al. (2020)

Familia CENTRISCIDAE Bonaparte, 1831

Macroramphosus scolopax (Linnaeus, 1758) Macroramphosus scolopax (Linnaeus, 
1758): Lozano Rey (1947); Fernández et al. (1978, 1981); SolórZANo et al. (1983, 1988); Rodríguez Villanueva \& VÁzQuez (1995); BAÑóN et al. (2010); BAÑóN \& SERRANO (2018)

\section{Macroramphosus gracilis (Lowe, 1839)}

Macroramphosus gracilis (Lowe, 1839): FARIÑA et al. (1985); Solórzano et al. (1983)

Comentarios: Durante un tiempo se consideraba que $M$. gracilis eran los juveniles de M. scolopax y por tanto la misma especie, si bien posteriormente se ha demostrado que son especies válidas y separadas. Debido a este criterio, $M$. gracilis no ha sido citada en las últimas revisiones de peces de Galicia.

Familia SYNGNATHIDAE Bonaparte, 1831

Hippocampus guttulatus Cuvier, 1829

Hippocampus guttulatus Cuvier, 1829: Lozano Rey (1919, 1947); Solórzano et al. (1988); BAÑón et al. (2010); BAÑóN \& SERRANO (2018)

Hippocampus ramulosus Leach, 1814: SoLÓRZANO et al. (1983); RODRÍGUEZ VILLANUEVA \& VÁzQuez (1995)

Hippocampus hippocampus (Linnaeus, 1758) Syngnathus hippocampus Linnaeus, 1758: CORNIDE (1788)

Hippocampus brevirostris Cuvier, 1829: López Seoane (1866); Paz Graells (1870)

Hippocampus antiquorum Leach, 1814: DE BUEN (1935b)

Hippocampus hippocampus (Linnaeus, 1758): Solórzano et al. (1983); VALLADARES et al. (2014); Bañón \& SERrano (2018)

Comentarios: Valladares et al. (2014) registran la presencia ocasional de $H$. hippocampus en Galicia. Si bien hay citas anteriores como sinonimias $H$. hippocampus (S. hippocampus, $H$. brevirostris y $H$. antiquorum), estas denominaciones se considera que corresponden a H. guttulatus (SolóRzANo et al. 1988; Rodríguez Villanueva \& VÁzQuez, 1995).

Entelurus aequoreus (Linnaeus, 1758)

Entelurus aequoreus (Linnaeus, 1758): DE Buen (1935b); Lozano Rey (1947); FARIÑA et al. (1985); SolóRZANo et al. (1983, 1988);
Rodríguez Villanueva \& Vázquez (1995); BAÑón et al. (2010, 2016a); BAÑón \& SERRANO (2018)

Nerophis lumbriciformis (Jenyns, 1835)

Nerophis lumbriciformis Fries: LozAno REY (1947)

Nerophis lumbriciformis (Jenyns, 1835): FARIÑa et al. (1985); SOlÓRZANo et al. (1983, 1988); Rodríguez Villanueva \& VÁzQuez (1995); BAÑón et al. (2010); BAÑóN \& SERRANO (2018)

Nerophis ophidion (Linnaeus, 1758)

Nerophis ophidion (Linnaeus, 1758): LÓPEZ SEOANe (1866); LozANo Rey (1947); FARIÑA et al. (1985); SOLÓRZANO et al. (1988); RODRÍGUEZ Villanueva \& VÁzquez (1995); BAÑón et al. (2010); Bañón \& Serrano (2018)

Syngnathus typhle Linnaeus, 1758

Syngnathus typhle Linnaeus, 1758: LóPEz SeoAne (1866); De Buen (1919, 1935b) Fariña et al. (1985); SolóRzano et al. (1983, 1988); Rodríguez Villanueva \& VÁzQuez (1995); BAÑón et al. (2010); BAÑón \& SERRANO (2018)

Syngnathus phlegon Risso, 1827

Syngnathus phlegon Risso, 1827: LozANo REY (1947); SolóRzANO et al. (1988); RodríGuez Villanueva \& VÁzquez (1995); BañóN et al. (2010); Bañón \& SERrano (2018)

Comentarios: Estatus dudoso. Especie típica del Mediterráneo, aunque LozAnO REY (1947) la cita frente a Marín, pero no se ha vuelto a encontrar.

Syngnathus acus Linnaeus, 1758

Siphostoma acus Rafinesque 1810: LóPEZ SEOANE (1866)

Syngnathus acus Linnaeus, 1758: De BUEN (1919, 1935b) FERNÁNDEZ et al. (1978); FARIÑA et al. (1985); Solórzano et al. (1983, 1988); Rodríguez Villanueva \& VÁzQuez (1995); BAÑón et al. (2010); BAÑón \& SERRANO (2018)

Syngnathus abaster Risso, 1827

Syngnathus pelagicus Linnaeus, 1758: LoZANO REY (1947)- error de identificación 
Syngnathus abaster Risso, 1827: SolóRZANO et al. (1988); Rodríguez VillanUeVA \& VÁzQuez (1995); BAÑón et al. (2010); BAÑóN \& Serrano (2018)

Comentarios: Lozano Rey (1947) cita $S$. pelagicus para Galicia, pero esta especie está distribuida en el Atlántico oeste y no está registrada en nuestras costas.

Syngnathus rostellatus Nilsson, 1855

Syngnathus rostellatus Nilsson, 1855: SolórzANo et al. (1983)

Comentarios: Estatus dudoso. Solórzano et al. (1983) la citan en O Grove, pero esta cita no ha sido incluida en las siguientes revisiones y actualizaciones.

\section{A}

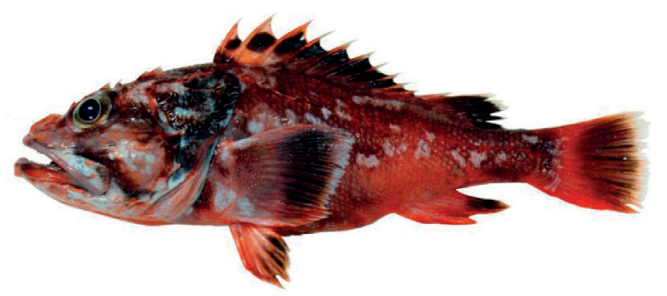

C

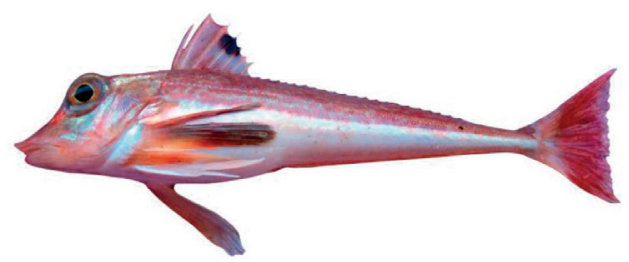

$\mathbf{E}$

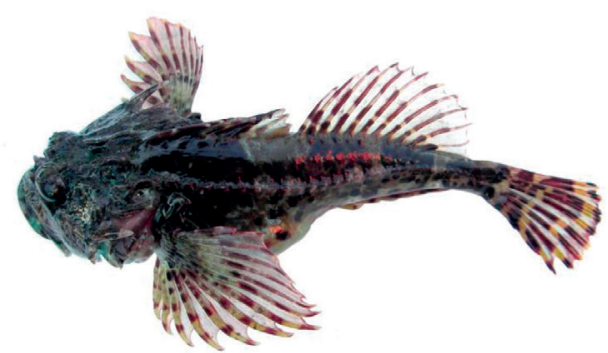

\section{Orden SCORPAENIFORMES} Familia SEBASTIDAE Kaup, 1873

Helicolenus dactylopterus (Delaroche, 1809) Scorpaena dactyloptera Delaroche, 1809: De Buen (1919)

Helicolenus dactylopterus (Delaroche, 1809): De Buen (1935b); Lozano Rey (1952); FERnÁNDEZ et al. (1978, 1981); FARIÑa et al. (1985); SOlÓRZANo et al. (1983, 1988); RodríGuez Villanueva \& VÁzQuez (1995); Piñeiro et al. (1996); BAÑÓN et al. (2010, 2016a); BAÑÓN \& Serrano (2018)

Trachyscorpia cristulata echinata (Koehler, 1896) (Fig. 5A)

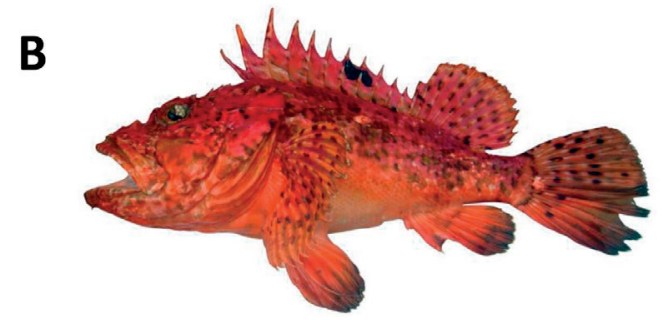

D

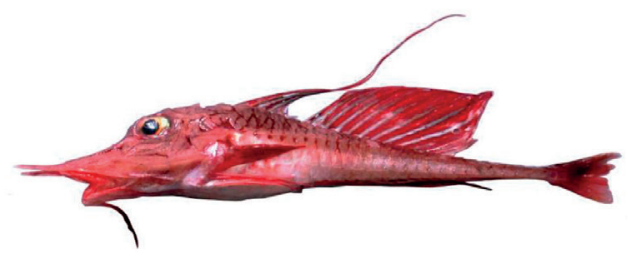

$\mathbf{F}$

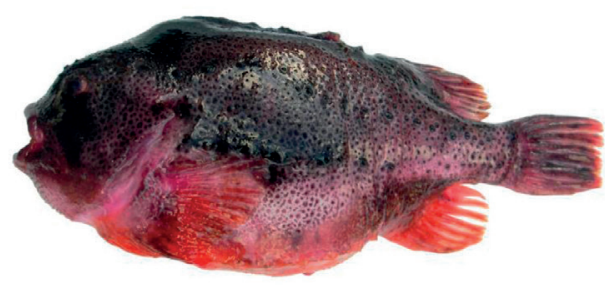

Figura 5. Peces Scorpaeniformes presentes en Galicia. (A) Trachyscorpia cristulata echinata (Koehler, 1896) (Sebastidae). (B) Scorpaena scrofa Linnaeus, 1758 (Scorpaenidae). (C) Eutrigla gurnardus (Linnaeus, 1758) (Triglidae). (D) Peristedion cataphractum (Linnaeus, 1758) (Peristediidae). (E) Taurulus bubalis (Euphrasen, 1786) (Cottidae). (F) Cyclopterus lumpus Linnaeus, 1758 (Cyclopteridae).

Figura 5. Scorpaeniform fishes in Galicia. (A) Trachyscorpia cristulata echinata (Koehler, 1896) (Sebastidae). (B) Scorpaena scrofa Linnaeus, 1758 (Scorpaenidae). (C) Eutrigla gurnardus (Linnaeus, 1758) (Triglidae). (D) Peristedion cataphractum (Linnaeus, 1758) (Peristediidae). (E) Taurulus bubalis (Euphrasen, 1786) (Cottidae). (F) Cyclopterus lumpus Linnaeus, 1758 (Cyclopteridae). 
Trachyscorpia cristulata echinata (Koehler, 1896): PiÑEIRO et al. (1996); BAÑón et al. (2010, 2016a); BAÑón \& SERRANo (2018)

Familia SCORPAENIDAE Risso, 1827

Pontinus kuhlii (Bowdich, 1825)

Pontinus kuhlii (Bowdich, 1825): FERNÁNDEZ et al. (1978); FARIÑA et al. (1985); SOLÓRZANo et al. (1988); RodRÍGUEz VillanUEVA \& VÁzQUEz (1995); BAÑón et al. (2010); BAÑóN \& Serrano (2018)

Scorpaena scrofa Linnaeus, 1758 (Fig. 5B)

Cottus scorpius Linnaeus, 1758: CORNIDE (1788)- error de identificación

Scorpaena scrofa Linnaeus, 1758: LóPEZ Seohne (1866) [Scorpaena scropha]; Paz Graells (1870); De Buen (1935b); Lozano REY (1952); FERNÁNDEZ et al. (1978); SOLÓRZANO et al. (1983, 1988); RodRÍGUEZ VILlanUEVA \& VÁzQUEz (1995); BAÑóN et al. (2010); BAÑóN \& Serrano (2018)

Comentarios: Cornide (1788) cita $C$. scorpius que Pérez ArCAs (1921) asigna a $S$. scrofa. Sin embargo, posteriores autores (SoLÓRZANO et al., 1998, RODRÍGUEZ VILLANUEVA \& VÁzQuEZ, 1995) asignan erróneamente a Cornide (1788) el nombre de Scorpius capito, omitiendo Cottus, el verdadero género que nombra Cornide (Cottus scorpius), y lo asignan a Taurulus bubalis. La descripción y el nombre común de "escarapote" confirman esta especie como una Scorpaena.

Scorpaena porcus Linnaeus, 1758

Scorpaena porcus Linnaeus, 1758: CORNIDE (1788); López Seoane (1866); Paz Graells (1870); De Buen (1919, 1935b); Lozano Rey (1952); FARIÑA et al. (1985); SolóRZANO et al. (1983, 1988); Rodríguez Villanueva \& VÁzQUEZ (1995); BAÑón et al. (2010); BAÑóN \& Serrano (2018)

Scorpaena notata Rafinesque, 1810

Scorpaena notata Rafinesque, 1810: DE Buen (1935b); Fernández et al. (1978); Solórzano et al. (1983, 1988); RodríGuez Villanueva \& VÁzQUez (1995); BAÑón et al. (2010); BAÑón \& SERRANo (2018)
Scorpaena ustulata Lowe, 1841: LozAno REY (1952)

Scorpaena loppei Cadenat, 1943

Scorpaena loppei Cadenat, 1943: BAÑón et al. (2010); BAÑón \& SERRANO (2018)

Familia TRIGLIDAE Rafinesque, 1815

Lepidotrigla cavillone (Lacepède, 1801)

Lepidotrigla cavillone (Lacepède, 1801): FARIÑA et al. (1985); SOlÓRZANO et al. (1983, 1988); Rodríguez Villanueva \& Vázquez (1995); BAÑón et al. (2010); BAÑón \& SERRANO (2018)

Comentarios: Hay dudas de si las citas de esta especie no se corresponden en realidad a Lepidotrigla dieuzeidei, ya que actualmente solo se han encontrado ejemplares de esta especie en Galicia.

Lepidotrigla dieuzeidei Blanc \& Hureau, 1973

Lepidotrigla dieuzeidei Blanc \& Hureau, 1973: BAÑóN (2004); BAÑón et al. (2010); Bañón \& Serrano (2018)

Trigla lyra Linnaeus, 1758

Trigla lyra Linnaeus, 1758: DE BUEN (1919, 1935b); Lozano Rey (1952); Fernández et al. (1978); FARIÑA et al. (1985); SolóRZANO et al. (1983, 1988); RodríGUEZ VillanUEVA \& VÁzQUEZ (1995); BAÑón et al. (2010); BAÑóN \& Serrano (2018)

Chelidonichthys obscurus (Walbaum 1792) Aspitrigla obscura Linnaeus, 1764: FERNÁNDEZ et al. (1978); FARIÑA et al. (1985); SOlórzano et al. (1983, 1988); RodríGUEZ Villanueva \& VÁzQuez (1995)

Chelidonichthys obscurus (Walbaum 1792): BAÑón et al. (2010); BAÑón \& SERrano (2018)

Chelidonichthys lucerna (Linnaeus, 1758)

Trigla hirundo Linnaeus, 1758: CORNIDE (1788); López SeoAne (1866); Lozano Rey (1952)

Trigla laevis Montagu, 1811: Paz Graells (1870)

Trigla corax Bonaparte, 1834: DE BuEN (1919) 
Trigla lucerna Linnaeus, 1758: DE BUEN (1935b); FernÁNDEZ et al. (1978); FARIÑA et al. (1985); Solórzano et al. (1983, 1988); Rodríguez Villanueva \& VÁzquez (1995)

Chelidonichthys lucerna (Linnaeus, 1758): BAÑón et al. (2010); BAÑón \& SERRANO (2018) 1788)

Chelidonichthys lastoviza (Bonnaterre,

Triglopurus lastoviza (Brünnich, 1768): FARIÑA et al. (1985); SolórZANO et al. (1983, 1988); Rodríguez VillanueVa \& VÁzQuez (1995)

Chelidonichthys lastoviza (Bonnaterre, 1788): BAÑón et al. (2010); BAÑóN \& SERRANO (2018)

Chelidonichthys cuculus (Linnaeus, 1758)

Trigla gurnardus Linnaeus, 1758: CORNIDE (1788)- error de identificación

Trigla cuculus Linnaeus, 1758: López Seoane (1866); Paz Graells (1870); De Buen (1935b); Lozano Rey (1952); Solórzano et al. (1983)

Aspitrigla cuculus (Linnaeus, 1758): FerNÁNDEZ et al. (1978, 1981); FARIÑA et al. (1985); SolóRZANo et al. (1988); BAÑón et al. (2010)

Chelidonichthys cuculus (Linnaeus, 1758): BAÑón \& SERRANO (2018)

Eutrigla gurnardus (Linnaeus, 1758) (Fig. 5C)

Cottus scaber Linnaeus, 1758: CoRNIDE (1788)- error de identificación

Trigla gurnardus Linnaeus, 1758: LóPEZ Seoane (1866); Paz Graells (1870); De Buen (1935b); LozANO REy (1952)

Eutrigla gurnardus (Linnaeus, 1758): FERNÁNDEZ et al. (1978, 1981); FARIÑA et al. (1985); SOLÓRZANO et al. (1983, 1988); RodRÍGuez Villanueva \& Vázquez (1995); Bañón et al. (2010); BAÑón \& SERRANo (2018)

Familia PERISTEDIIDAE Jordan \& Gilbert, 1883

Peristedion cataphractum (Linnaeus, 1758) (Fig. 5D)

Peristedion cataphractum (Linnaeus, 1758): Solórzano et al. (1983, 1988); RodríGUEZ Villanueva \& VÁzQuez (1995); Bañón et al. (2010); BAÑón \& SERRANo (2018)
Familia COTTIDAE Bonaparte, 1831

Taurulus bubalis (Euphrasen, 1786) (Fig. 5E) Cottus scorpius Linnaeus, 1758: DE BuEN (1919)- error de identificación

Cottus bubalis Euphrasen, 1786: LozAno REY (1928, 1952)

Acanthocottus bubalis (Euphrasen, 1786): De Buen (1935b)

Taurulus bubalis (Euphrasen, 1786): FARIÑA et al. (1985); SOLÓRZANO et al. (1983, 1988); Rodríguez Villanueva \& VÁzQuez (1995); BAÑón et al. (2010); BAÑón \& SERrano (2018)

Comentarios: De Buen (1919) asigna el nombre de $C$. scorpius a T. bubalis, pero C. scorpius es considerado un sinónimo de Myoxocephalus scorpius (Linnaeus, 1758) (WORMS, 2020), otra especie de la misma familia pero ausente en nuestras costas.

Familia PSYCHROLUTIDAE Günther, 1861

Cottunculus thomsonii (Günther, 1882)

Cottunculus thomsonii (Günther, 1882): PiñeIro et al. (1996); BAÑón et al. (2010); Bañón \& Serrano (2018)

Familia CYCLOPTERIDAE Bonaparte, 1831

Cyclopterus lumpus Linnaeus, 1758 (Fig. 5F)

Cyclopterus lumpus Linnaeus, 1758: SolóRZANO et al. (1983, 1988); Rodríguez VillaNUEVA \& VÁzQUEz (1995); BAÑón et al. (2008b, 2010); BAÑón \& Serrano (2018)

Comentarios: Especie boreal, ocasional, con presencia estacional, principalmente en primavera, cuando se acerca a nuestras costas para reproducirse (BAÑón et al., 2008b)

Familia LIPARIDAE Gill, 1861

Liparis montagui (Donovan, 1804)

Liparis montagui (Donovan, 1804): DevesA \& Solórzano (1983); Solórzano et al. (1983, 1988); Rodríguez Villanueva \& Vázquez (1995); BAÑón et al. (2008); BAÑón et al. (2010); Bañón \& SERrano (2018)

Paraliparis membranaceus Günther, 1887 Paraliparis membranaceus Günther, 1887: BAÑón et al. (2010); BAÑón \& SERRANO (2018) 
Paraliparis hystrix Merret, 1983

Paraliparis hystrix Merret, 1983: BAÑón et al. (2016a); Bañón \& Serrano (2018)

\section{DISCUSIÓN}

La revisión taxonómica de los peces de Galicia en función de su nomenclatura científica nos permite validar o no la identificación de cada autor, detectar errores de identificación y conocer la evolución histórica de la ictiología marina de Galicia.

Detectar los errores de identificación, especialmente en los primeros autores, es una labor compleja, dada la ausencia o escasez de información en la bibliografía que permita identificar la especie. Un ejemplo es el caballito de mar H. hippocampus, citado en Galicia en 2010 (VALLADARES et al., 2014), si bien hay citas dudosas anteriores. CORNIDE (1788), López Seodne (1866) y De Buen (1935b) citan $S$. hippocampus, $H$. brevirostris y $H$. antiquorum respectivamente, que son considerados sinónimos de H. hippocampus (Worms, 2020). Sin embargo, dado que la especie más abundante actualmente y supuestamente también en el pasado es $H$. guttulatus, hay dudas de si se trata de un error de identificación, es decir, si se asignó erróneamente a $H$. guttulatus el nombre científico de $H$. hippocampus, como apuntan SolóRZANO et al. (1988) y RoDRÍGUEZ Villanueva \& VÁzQuez (1995) para el caso de $H$. antiquorum, o si, por el contrario, son identificaciones válidas de $H$. hippocampus. PÉrez ArCas (1921) sugiere que con el nombre de $H$. brevirostris se nombran indistintamente a las dos especies, que es posiblemente lo que sucedió, por lo que resulta imposible saber a cual de las dos especies se refiere cada autor.

En anteriores revisiones de la ictiofauna gallega (Solórzano et al. 1983, 1988; RodRíGuez Villanueva \& VÁzQuez, 1995), las primeras fuentes bibliográficas son poco citadas, probablemente por desconocimiento. En la actualidad, muchos de estos tratados (López Seoane, 1866; De Buen, 1919; Lozano Rey, 1919) se han digitalizado, y son de fácil acceso a los investigadores, lo cual nos ha permitido completar el conocimiento existente en las primeras etapas de la ictiología de Galicia y adelantar considerablemente la primera cita de algunas especies con respecto a anteriores revisiones. Conocer el año de la primera cita es importante no solo a nivel historiográfico, sino también a nivel biogeográfico, biológico y ecológico.

Otra fuente bibliográfica menos conocida pero de gran importancia es la cita de especies de Galicia fuera de España. De Buen (1936) y LozAno Rey $(1947,1952,1960)$ ya recogen citas de especies capturadas frente a Galicia por investigadores foráneos como Legendre, Roule, Vaillant, Steindachner o Zugmayer. La mayoría de las citas corresponden a investigadores franceses, dada la relativa cercanía de nuestras costas y a que Francia cuenta con una tradición de investigación ictiológica mayor que la de España. Además, antes de la extensión a 200 millas de la zona económica exclusiva en 1978, los barcos franceses, tanto comerciales como de investigación, trabajaban frecuentemente frente a nuestras costas, capturando especies que luego eran estudiadas y publicadas en revistas científicas de aquel país. De ahí proceden, por ejemplo, las primeras citas para Galicia de G. brachiusculus (Quéro, 1970), Z. conchifer (Quéro, 1972), B. splendens y B. decadactylus (CLIGNY, 1903), M. poutassou y H. mediterraneus (CLIGNY, 1905).

Finalmente, la revisión bibliográfica nos ha permitido añadir al listado de peces de Galicia nuevas especies que antes habían pasado desapercibidas, como $S$. ampullaceus, $L$. sphyrenoides, A. pharao, D. luetkeni, L. alatus, $L$. gemellarii y C. pinnatibarbatus (DE BUEN, 1936).

\section{REFERENCIAS}

Anadón, E. (1957). Anatomía e histología de las placas luminosas caudales de Lampadena nitida (Taaning). Boletín de la Real Sociedad Española de Historia Natural Sección biológica, 55(1): 129-144.

Arté, P (1952a). Peces raros de los grandes fondos de las aguas gallegas. Industrias Pesqueras, 625: 48-51.

Arté, P. (1952b). Notas ictiologicas. I. Peces raros o nuevos para el literal gallego (N.W. de España). Publicaciones del Instituto de Biologia Aplicada Barcelona, 10, 93-103. 
Bañón, R., Casas, J.m., Piñeiro, C.g. \& Covelo, M. (1997). Capturas de peces de afinidades tropicales en aguas atlánticas de Galicia (NO de la península Ibérica). Boletín del Instituto. Español de Oceanografía, 13 (1 у 2): 57-66.

BAÑón R. (2002). Actualización del listado faunístico de peces de mar de Galicia. Nova Acta Científica Compostelana (Bioloxía), 12: 119-123.

Bañón, R., Del Río, J.l., Piñeiro, C. \& Casas, M. (2002). Occurrence of tropical affinity fishes in Galician waters NW Spain. Journal of the Marine Biological Association of the United Kingdom, 82: 877-880. https://doi. org/10.1017/S0025315402006288

Bañón, R., Armesto, A. (2003). Nota sobre un ejemplar de rape (Lophius budegassa Spinola, 1807) (Lophiiformes: Lophiidae) con coloración atípica. Nova Acta Científica Compostelana (Bioloxía), 13: 107-109.

BAÑón, R. (2004). New records of two Southern fish in Galician waters (NW Spain). Cybium, 28 (4): 367-368.

Bañón, R., Pietsch, T.w., Piñeiro, C.g. (2006). New record of Linophryne coronata, (Lophiiformes, Linophrynidae) from the North-eastern Atlantic Ocean. Cybium, 30 (4): 385-386.

Bañón, R., Cerdeira, J.d., Ferreiro, P. \& Sande, C.M. (2007). Nuevas aportaciones a la ictiofauna marina de Galicia. Nova Acta Científica Compostelana (Bioloxía), 16, 161-163.

BAÑón, R \& SANDE, C. (2008). First record of the cornetfish Fistularia petimba (Syngnathiformes: Fistularidae) from Galician waters. A northernmost occurrence in the eastern Atlantic. Journal of Applied Ichthyology, 24: 106-107. https://doi.org/10.1111/j.14390426.2007.00918.x

Bañón, R., Ferreiro, P., García-Seoane, E., Oliver, P. \& Ínsua, J. (2008a). Nuevos datos sobre la presencia de especies nuevas o poco conocidas de la ictiofauna marina de Galicia (II). Nova Acta Cientifica Compostelana (Bioloxía), 17: 191-194.

Bañón, R., Garazo, A. \& Fernández, A. (2008b). Note about the presence of the lumpsucker Cyclopterus lumpus (Teleostei, Cyclopteridae) in Galician waters (NW Spain). Journal of Applied Ichthyology, 24:
108-109. http://dx.doi.org/10.1111/j.14390426.2007.00839.x

Bañon, R., Villegas-Ríos, D., Serrano, A., Mucientes, G. \& Arronte, J.C. (2010). Marine fishes from Galicia (NW Spain): an updated checklist. Zootaxa, 2667, 1-27. https://doi.org/10.11646/zootaxa.2667.1.1

Bañón, R., Valeiras, X., Armesto, A.\& Abad, E. (2011a). Occurrence of Ophisurus serpens (Anguilliformes, Ophichthidae) from Galician waters (NW Spain). Cybium, 35 (2): 157-158.

Bañón, R., Arronte, J.c., Serrano, A. \& SÁNCHEZ, F. (2011b). First records of Purplemouthed conger Pseudophichthys splendens (Anguilliformes: Congridae) from the Galicia Bank (NW Spain). A northward range extension of their distribution in the eastern Atlantic. Cybium, 35 (3): 262-264.

Bañón, R., Arronte, J.C., VÁzquez-Dorado, S., Del Río, J.l. \& De Carlos, A. (2013). DNA barcoding of the genus Lepidion (Gadiformes: Moridae) with recognition of Lepidion eques as a junior synonym of Lepidion lepidion. Molecular Ecology Resources, 13: 189-199. https://doi.org/10.1111/17550998.12045

Bañón, R., Arronte, J.c., Barros García, D., Vázquez Dorado, S. \& De Carlos, A. (2013). Taxonomic study of Bathygadidae fishes (Gadiformes) from Atlantic Spanish waters combining morphological and molecular approaches. Zootaxa, 3746 (4): 552-566. http://dx.doi.org/10.11646/zootaxa.3746.4.3

Bañon, R., Arronte, J.c., Isbert, W., Coscelli, G. \& SAnchez, F. (2014). Melanic hyper-pigmentation in the genus Lepidion (Gadiformes: Moridae). Cybium, 38 (3): 231-234.

Bañón, R., Arronte, J.c., Rodríguez-Cabello, C., Piñeiro, C.g., Punzón, A. \& Serrano, A. (2016a) Commented checklist of marine fishes from the Galicia Bank seamount (NW Spain). Zootaxa, 4067: 293-333. https://doi. org/10.11646/zootaxa.4067.3.2

Bañón, R., Arronte, J.c., Barros García, D. \& De Carlos, A. (2016b). Halosaur fishes (Notacanthiformes: Halosauridae) from Atlantic Spanish waters according to integrative taxonomy. Zootaxa 4184(3): 471-490. http:// dx.doi.org/10.11646/zootaxa.4184.3.3 
Bañón, R., Punzón, A., Barros García, D. \& De Carlos, A. (2019). Range extension of the Atlantic herring Clupea harengus (Clupeiformes: Clupeidae) southern part of the Northeast Atlantic Ocean. Cybium, 43(3): 291-293. https://doi.org/10.26028/ cybium/2019-433-011

Bañón, R., Tejerina, R., Morales, X., Alonso-Fernández, A., Barros-García, D., De CARlos, A. (2019). Unusual occurrences of fishes along the Northeast Atlantic: new biological and distributional data. Mediterranean Marine Science, 20 (1): 189-196. https:// doi.org/10.12681/mms. 19307

Bañón, R., Caballero-Huertas, M., AlonsoFernández, A., Barros-García, D., Otero, J., De Carlos, A. (2020). Signals of changes in the ich thyofauna composition of Galician waters (NW Iberian Peninsula). Regional Studies in Marine Science, 101435. https:// doi.org/10.1016/j.rsma.2020.101435

Barros García, D., Bañón, R., Arronte, J.c., Fernández-Peralta, L., García, R. \& De Carlos, A. (2016A). Dna barcoding of deep-water notacanthiform fishes (Teleostei: Elopomorpha). Zoologica Scripta, 45 (3): 263-272. http://dx.doi.org/10.111/zsc.12154

Barros García, D., Bañón, R., Arronte, J.c. \& De CArlos, A. (2016b). New data reinforcing the taxonomic status of Lepidion eques as synonym of Lepidion lepidion (Teleostei, Gadiformes). Biochemical Systematics and Ecology, 68:6-10. http://dx.doi.org/10.1016/j. bse.2016.06.017

Barros García, D., Bañón, R., Arronte, J.c., Fernández-Peralta, L., García, R., Iglesias, S., Sellos, D., Barreiros, J.P., Sebastián-Comesaña, A. \& De Carlos, A. (2018). New insights into the systematics of North Atlantic Gaidropsarus (Gadiformes, Gadidae): flagging synonymies and hidden diversity. Marine Biology Research, 14(1): 17-29. https://doi.org/10.1080/17451000.20 17.1367403

Barros García, D., Froufe, E., Bañón, R., Arronte, J.c., Baldó, F., De Carlos, A. (2020). Phylogeography highlights two different Atlantic/Mediterranean lineages and a phenotypic latitudinal gradient for the
Deep-Sea Morid Codling Lepidion lepidion (Gadiformes: Moridae). Deep-Sea Research Part I, 157, 103212. https://doi.org/10.1016/j. dsr.2019.103212

Barros García, D., Gomes Dos Santos, A., Machado, A., Castro, F.c., De Carlos, A., Bañón, R., Bruno, I., Arronte, J.c., Froufe, E. (2020). Complete mitogenome of the shortfin spiny eel, Notacanthus bonaparte (Elopomorpha; Notacanthiformes). Mitochondrial DNA Part B, 5(2): 1421-1422. https://doi.org $/ 10.1080 / 23802359.2020 .1736672$

CADÉE, G.C. (1968). Molluscan biocoenoses and thanatocoenoses in the Ría de Arosa, Galicia, Spain. Zoologische Verhandelingen, 95: 1-121.

Carneiro, M., Martins, R., Reiner, F. \& BaTISTA, I. (2019). Ichthyofauna of Portugal: Taxonomic diversity, common and scientific names of marine fishes. IPMA, I.P., 1, $376 \mathrm{p}$.

Cligny, A. (1903). Poissons des côtes d'Espagne et Portugal (Océan Atlantique). Prémier partie. Annales de la Station aquicole de Boulogne-sur-mer, 1: 1-30

Clingy, A. (1905). Poissons des côtes d'Espagne et du Portugal(Océan Atlantique), deuxième partie. Annales de la Station aquicole de Boulogne-sur-mer., 1: 69-92.

Cornide, J. (1788). Ensayo de una historia de los peces y otras producciones marinas de la costa de Galicia. Edición facsímil, estudio preliminar por V. Paz-Andrade. Edición do Castro, O Castro-Sada. 1983. 263 pp.

De Buen, O. (1919). Mr. Xavier Ducloux y sus trabajos sobre peces y pesca en Vigo. Boletín de Pescas, 31-32: 81-83.

De Buen, F. (1935a). Fauna ictiológica. Catálogo de los peces ibéricos de la planicie continental, aguas dulces, pelágicos y de los abismos próximos. I parte. Notas y Resúmenes Instituto Español de Oceanografía, 82, 1-89.

De Buen, F. (1935b). Fauna ictiológica. Catálogo de los peces ibéricos de la planicie continental, aguas dulces, pelágicos y de los abismos próximos. II parte. Notas y Resúmenes Instituto Español de Oceanografía, 82, 89-148.

De Buen, F. (1936). Fauna Ictiologica, Catalogo de los peces ibericos: de la planicie continental, aguas dulces, pelagicos y de los abismos 
proximos. Tercera parte. Notas y Resumenes Instituto Espanol de Oceanografia Madrid, 94, 151-173.

De Carlos, A., Bañón, R., Cobo-Arroyo, S., Arronte, J.C., Del Río, J.L., Barros-García, D. (2020). DNA barcoding flags the existence of sympatric cryptic species in the slender codling Halargyreus johnsonii (Gadiformes, Moridae). Marine Biodiversity, 50, 49. https:// doi.org/10.1007/s12526-020-01074-8

Devesa, S. \& Solórzano, M.r. (1983). Aportaciones para un mejor conocimiento de la ictiofauna del litoral gallego. Cuadernos de Inice. Ciencias, 6, 37-45.

Fariña, A.c., Pereiro, F.J., Fernández A. (1985). Peces de los fondos de arrastre de la plataforma continental de Galicia. Boletín del Instituto Español de Oceanografía, 2(3): 89-98.

Fariña, A.C., Pérez, A. \& Morlán, R. (1999). First record of Neocyttus helgae (Pisces: Oreosomatidae) on the slope off Galicia (Northwet Spain). Scientia Marina, 63(2): 177-180.

Fernández, A., Pereiro, F.X., Iglesias, S., Porteiro, C.\& Pallarés, P. (1978). La Pesquería demersal gallega, estrategias de pesca para su regulación racional en base a la Merluza. Boletín del Instituto. Españolde Oceanografía, 4 (249): 69-109.

FERnÁndeZ-CordeIro, A. (1997). Fifteen-spined Stickleback (Spinachia spinachia Linnaeus, 1758 ) in waters off the NW Iberian peninsula, southern limit of its range. Miscelania Zoologica, 20(1): 65-72.

Ferreira, E. (1998). O desenvolvemento da actividade pesqueira dende a Alta Idade Media ó século XVII. En: Historia da pesca en Galicia. Biblioteca de divulgación, Universidade de Santiago de Compostela, p. 51-75.

Fidalgo, X.A. (1998). Arredor do polbo. Do mar á montaña. En: Antropoloxía mariñeira. Actas do Simposio Internacional de Antropoloxía in memorian Xosé Filgueira Valverde(F. CAlo Lourido coord.). Santiago de Compostela. Consello da Cultura Galega. Pp. 195-228.

Franco, C. \& Sola, A. (1985). Estudios de ictioplancton de especies de interés comercial de la plataforma gallega. Boletín del Instituto Español de Oceanografía, 2(3): 15-26.
Fricke, R., Eschmeyer, W.n. \& VAn Der LAAN R. (2020). Eschmeyer's Catalog Of Fishes: Genera, Species, References.(http:// researcharchive.calacademy.org/research/ ichthyology/catalog/fishcatmain.asp). Electronic version accessed 20 April 2020.

GonzÁlez, E. (2013). La ictiofauna de los yacimientos arqueológicos del noroeste de la Península Ibérica. Tesis doctoral, Universidad de León. 471 pp.

GAEMERs. P.A.M. (2016). Taxonomy, distribution and evolution of Trisopterine Gadidae by means of otoliths and other characteristics. Fishes, 1: 18-52. http://dx.doi.org/10.3390/ fishes 1010018

Henriques, M., Lourenço, R., Almada, F., Calado, G., Gonçalves, D., Guillemaud, T., Cancela, M.L. \& Almada, V.c. (2002). A revision of the status of Lepadogaster lepadogaster (Teleostei: Gobiesocidae): sympatric subspecies or a long misunderstood blend of species? Biological Journal of the Linnean Society, 76: 327-338.

IGLESIAS, J. (1981). Spatial and temporal changes in the demersal fish community of the Ría de Arosa (NW Spain). Marine Biology, 65: 199-208.

Juega Puig, J. (2012). El comercio marítimo en Galicia, 1525-1640. Obradoiro de historia moderna, 21: 105-130.

López SeoAne, V. (1866). Reseña de la historia natural de Galicia. Edición facsímil, Edición do Castro, 1996. Sada, A Coruña. 66 pp.

LoZANO REy (1919). Los peces de la fauna ibérica en la colección del museo en 1 de enero de 1919. Trabajos del Museo Nacional de Ciencias Naturales. Serie Zoológica, 39: 1-122.

Lozano Rey, L. (1947). Peces Ganoideos y Fisóstomos. Memorias de la Real Academia de ciencias exactas, físicas y naturales. Serie de Ciencias Naturales ; tomo XIV. Real Academia de ciencias exactas, físicas y naturales, Madrid, XI, 839 p.

Lozano Rey, L. (1952). Peces fisoclistos, Subserie Toracicos. Memorias de la Real Academia de Ciencias Exactas, Fisicas y Naturales de Madrid. Serie Ciencias Naturales., Primera parte, 14, 1-378; Segunda parte, 14, 379-705. 
Lozano Rey, L. (1960). Peces fisoclistos, Subserie Toracicos Ordenes Equeneiformes y Gobiformes), Pediculados y asimétricos. Memorias de la Real Academia de Ciencias Exactas, Fisicas y Naturales de Madrid. Serie Ciencias Naturales., Tercera parte, 14: 1-613.

López VeigA, E.c. (1976). Aspectos de la reproducción y maduración sexual del espadín (Sprattus sprattus L.) de Galicia. Investigación Pesquera, 40: 95-104.

Lopez VeigA, E.c (1978). Parametros poblacionales de la sardina (Sardina pilchardus) de Galicia. Investigación Pesquera, 42: 335-339.

Navaz Y Sanz, J. M. (1946). Sobre algunos peces poco frecuentes o desconocidos en las costas de Galicia. Notas y Resúmenes del Instituto Español de Oceanografía, 133, 1- 7.

Oliver, M. (1950). Estudios sobre el Espadín (Clupea sprattus L.) de la costa Noroeste de España. Boletín del Instituto Español de Oceanografia, 26: 1-16.

Paz Graells, M. (1870). Exploración científica de las costas del departamento marítimo de Ferrol verificada de orden del Almirantazgo por el vocal de la Comisión Permanente de Pesca D. Mariano de la Paz Graells en el verano de 1869. Impr. Fortanet, Madrid. 540 pp.

PÉrez ArCAs, L. (1921). Ictiología Ibérica, o sea Catálogo de los peces marinos y de agua dulce que habitan o frecuentan las costas de la Península Ibérica. Revista de la Real Academia de Ciencias Exactas, Físicas y Naturales de Madrid. Tomo XIX: $4^{\mathrm{o}}$ de la $2^{\mathrm{a}}$ Serie: 24-548.

Piñeiro, C., Casas, J.m., Bañón, R., Serrano, A. \& Calviño, A. (1996). Resultados de la Acción piloto de pesca experimental en el Talud de la Plataforma Gallega (Noroeste de la Península Ibérica). Datos y Resúmenes del Instituto Español de Oceanografía, 2, 1-57.

Punzón, A. \& Serrano, A. (1998). First record of Ijimaia loppei Roule, 1922 (Lampriformes: Ateleopododidae) in the Iberian Peninsula waters. Cybium, 22(3): 297-299.

QuÉRO, J.C. (1970). Observations françaises sur les poissons rares en 1968 et 1969 . Conseil Permanent International pour l'Exploration de la Mer, Annales Biologiques, 26: 280-282. QuÉRo J.C. (1972). Observations françaises sur les poissons rares en 1970. Conseil Permanent
International pour l'Exploration de la Mer, Annales Biologiques, 27: 195.

Quéro, J.c. \& Robles, R. (1977). Captures de Zeides (Pisces, Zeiforme) dans l'Atlantique est au nord de $40^{\circ}$ N. Cybium, 2: 107-113.

QuÉro, J.C. (1979). Remarques sur le Grammicolepis brachiusculus (Pisces, Zeiformes, Grammicolepididae). Annales de la Société des sciences naturelles de la Charente-Maritime, 6 (6): 573-576.

Rodríguez Villanueva, X.l. \& Vázquez, X. (1994). Peixes do Mar de Galicia (II). Peixes óseos: xeneralidades, clasificación e orde perciformes. Colección Montes e Fontes. Ed. Xerais de Galicia, 351 pp.

Rodríguez Villanueva, X.L. \& Vázquez, X. (1995). Peixes do Mar de Galicia (III). Peixes óseos (continuación). Colección Montes e Fontes. Ed. Xerais de Galicia, 415 pp.

SÁÑez Reguart, A. (1796). Colección de producciones de los mares de España. (Isabel García Fajardo, J. Fernández Pérez, Eds.). Ministerio de Agricultura, Pesca y Alimentación, Secretaría General Técnica, Madrid. 1993.

Sarmiento, M. (1762-1766). Obra llamada de 660 pliegos que trata de historia natural y de todo género de erudición. Copia manuscrita, vol2. Biblioteca Digital Hispánica, en http:// bdh-rd.bne.es/viewer.vm?id=0000038853

Solórzano, M.r., Devesa, S. \& Soutullo, L. (1983). Guía dos peixes de Galicia. Ed. Galaxia, Vigo, $224 \mathrm{p}$.

Solórzano, M.R., Rodríguez, J.L., Iglesias, J., Pereiro, F.x. \& Álvarez, F. (1988). Inventario dos Peixes do Litoral Galego (Pisces: Cyclostomata, Chondrichthyes, Osteichthyes). Cadernos da área de Ciencias Biolóxicas, (Inventarios). Seminario de Estudos Galegos, Vol IV. Ed. Do Castro, O Castro-Sada, A Coruña. 69 pp.

Valladares, S., Bañón, R., López, A., Bouza, C., Chamorro, A., García, M.e. \& Planas, M. (2014). First records of the seahorse Hippocampus hippocampus in Galician waters (NW Spain). Cybium, 38 (1): 74-76.

VAN Der LAAn, R., Eschmeyer, W. N. \& Fricke, R. (2014). Family-group names of Recent 
fishes. Zootaxa Monograph, 3882 (1), 1-230. http://dx.doi.org/10.11646/zootaxa.3882.1.1 Worms (2020). World Register of Marine Species. Available from http://www.marine- species.org at VLIZ. Accessed 2020-03-29. https://doi.org/10.14284/170 